\title{
NEW BACTERIA FORAGING AND PARTICLE SWARM HYBRID ALGORITHM FOR MEDICAL IMAGE COMPRESSION
}

\author{
G. Vimala KUMARI ${ }^{\bowtie, 1}$, G. SASIBHUShana RaO ${ }^{2}$, B. PrabHaKARA RAO ${ }^{3}$ \\ ${ }^{1}$ Department of Electronics and Communication Engineering, M.V.G.R.College of Engineering, Vizianagaram- \\ 535005, India; ${ }^{2}$ Andhra University College of Engineering, Visakhapatnam-530003, India; ${ }^{3}$ Jawaharlal Nehru \\ Technological University, Kakinada, Kakinada-533003, Andhra Pradesh, India \\ e-mail: vimalakumari7@gmail.com, sasigps@gmail.com,drbprjntuk@gmail.com \\ (Received November 25, 2017; revised May 29; 2018; accepted September 9, 2018)
}

\begin{abstract}
For perfect diagnosis of brain tumour, it is necessary to identify tumour affected regions in the brain in Magnetic Resonance Imaging (MRI) images effectively and compression of these images for transmission over a communication channel at high speed with better visual quality to the experts. An attempt has been made in this paper for identifying tumour regions with optimal thresholds which are optimized with the proposed Hybrid Bacteria Foraging Optimization Algorithm (BFOA) and Particle Swarm Optimization (PSO) named (HBFOA-PSO) by maximizing the Renyi's entropy and Kapur's entropy. BFOA may be trapped into local optimal problem and delay in execution time (convergence time) because of random chemotaxis steps in the procedure of algorithm and to get global solution, a theory of swarming is commenced in the structure of HBFOA-PSO. Effectiveness of this HBFOA-PSO is evaluated on six different MRI images of brain with tumours and proved to be better in Peak Signal to Noise Ratio (PSNR), Mean Square Error (MSE) and Fitness Function.
\end{abstract}

Keywords: Bacteria Foraging Optimization Algorithm (BFOA), Grey Wolf Optimization (GWO), Kapur's entropy, Moth-flame Optimization (MFO) Algorithm, Particle Swarm Optimization (PSO), Renyi’s entropy.

\section{INTRODUCTION}

In this modern world human beings are very much busy with their personal and professional life and undisciplined food and sleeping timings and busy life style which are causing health disorders. Brain tumour is one such dangerous health disorder and it is due to growth of cells abnormally in the tissues of the brain and can directly destroy or damage brain cells by producing inflammation. Brain tumours are classified by their size and type of tissue involved. In the areas of human brain image analysis, recognition of tumour region and segmentation of tissue organization tend to be a demanding task. Computerized segmentation of Magnetic Resonance (MR) brain images would be of immense help to radiologists, as they reduce the difficulties developed due to human interface and offer quicker segmentation results. Computerized algorithms offer negligible time duration and slighter manual involvement to a radiologist during clinical diagnosis. In addition, huge volumes of patients' information can be evaluated by computerization. For this purpose one algorithm called Hybrid Bacteria Foraging Optimization
Algorithm and Particle Swarm Optimization (HBFOAPSO) is proposed for effective and efficient image segmentation for identification of the brain tumour and segmented image is transmitted over a communication channel after successful compression and is received at the receiver section by a radiologist for diagnosis of brain tumour and for necessary action. This process is called telemedicine and its main objective is to provide clinical care from a distance. This benefits many rural areas and also at times of emergencies when doctor's presence is essential. But further development of this technology is becoming slow due to the limited narrow transmission bandwidths. As the presence and development of such technologies are crucial, a lot of research is being carried out for further improvement in effective usage of such technology. So in this paper, an HBFOA-PSO based image thresholding by maximizing Renyi's entropy and Kapur's entropy is proposed for efficient and effective results of image thresholding in better image compression, which helps reduce the Bit rate for transmission as well as maintains an appreciable amount of quality or fidelity of the image. Image thresholding is a process of optimizing similar regions 
in an image which results in effective clustering of image, hence better image compression is achieved by running cascaded runlength coding and arithmetic coding on cluster image. Performance of the runlength coding and arithmetic coding depends upon the number of similar clusters and probability occurrence of same cluster centroids in an image respectively. So, its performance depends upon effective clustering technique.

\section{MATERIAL AND METHODS}

\section{CONTRIBUTION}

In this section a detailed description is given about the methods of MRI image tumour recognitions and methods to compress the identified regions at high bit rate with better reconstructed image quality. In first section, methods of MRI image segmentation are explained and in second section methods of image compression are explained.

\section{RELATED WORK IN TUMOUR IDENTIFICATION}

Stochastic threshold of MRI image for tumour identification is done by combining region based level sets globally and three established energies (uniform, separation, and histogram) in a local framework (Lubna et al., 2017). An automated brain tumour threshold model based on maximum a posteriori probabilistic (MAP) estimation and likelihood probability of the model is estimated by sparse coding and dictionary learning. The Markov random field (MRF) is introduced into the prior probability. The MAP is converted into a minimum energy optimization problem and graph cuts are used to find its solution (Yuhong et al., 2016). Irem et al., proposed a Principal Component Analysis (PCA) based K-means and fuzzy C-means (FCM) clustering for MRI image thresholding and results are compared with five PCA algorithms such as PCA, Probabilistic Principal Component Analysis (PPCA), Expectation Maximization Based PCA (EM-PCA), Generalize Hebbian Algorithm (GHA), and Adaptive Principal Component Extraction (APEX) and proved EM-PCA and PPCA results are effective with the two clustering algorithms (Irem et al., 2017). The brain tumours are identified and marked with a novel technique which is proposed by solmaz (Solmaz and Farshad, 2017). The 3D images are pre-processed with the help of cascaded histogram matching and bias field correction. After this the required areas are extracted from background. Local binary pattern and orientation gradients are used for learning. Potential Field Clustering (PFS) is one which is based on concept of potential field and evaluates the performance of the different methods on the brain tumour MRI benchmark database (Ivan and Iker, 2017). Statistical fusion based image thresholding is proposed for identification of abnormalities in MRI images by seed selection, region growing and image fusion. The proposed technique is tested for performance analysis on different data base and different tumour effected images (Badri et al., 2016). Nooshin and Miroslav proposed automatic tumour thresholding in singlespectral MRI using a texture-based and contour-based algorithm (Nooshin and Miroslav, 2017).

Sudip et al., proposed a conditional spatial fuzzy Cmeans ( $\mathrm{csFCM}$ ) clustering algorithm for thresholding of MRI images by incorporating local and global spatial information into a weighted membership function and results are compared and proved better than K-means and FCM algorithms in terms of validity functions, threshold accuracy, tissue threshold accuracy, and receiver operating characteristic (ROC) curve (Sudip et al., 2015). Edge detection of X-ray images using Multiresolution Analysis (MRA) based biorthogonal wavelets is more preferable when compared with orthogonal wavelets because of more flexibility (GS Rao et al., 2016). Brain tumour is identified with efficient thresholding of MRI images by Bacteria Foraging Optimization (BFO) with modified fuzzy Kmeans algorithm (MFKM) and results are compared with Particle Swarm Optimization (PSO) based fuzzy C-means algorithm (PSO based FCM), MFKM and conventional FCM and proved better in terms of sensitivity, specificity, Jaccard Tanimoto Co-efficient index (TC) and Dice Overlap Index (DOI), computational time and memory requirement (Anitha et al., 2017). Computed Tomography (CT) images are classified by Support Vector Machine (SVM) with different kernel functions and Sequential Minimal Optimization (SMO) and segmented classification is further performed by the Modified Region Growing (MRG) with threshold optimization. These thresholds are optimized with Harmony Search (HS), Evolutionary Programming, (EP) and Grey Wolf Optimization (GWO). In terms of sensitivity, specificity, and accuracy GWO is better compared to others (Ramakrishnan and Sankaragomathi, 2017). MRI images are segmented with Teaching Learning Based Optimization (TLBO), entropy value, and level set/active contour and TLBO achieved better values in Jaccard index, dice co-efficient, precision, sensitivity, specificity, and accuracy (Rajinikanth et al., 2017). Crow Search Algorithm (CSA) based image thresholding by maximizing cross entropy for MRI image threshold and proved better with other techniques in terms of quality and consistency (Diego et al., 2017). 
Sathya and Kayalvizhi took Kapur's and Otsu's entropies as an objective functions and which are optimized with Bacterial Foraging Optimization Algorithm (BFOA) for effective and efficient image thresholding (Sathya and Kayalvizhi, 2011). Further, in order to improve the convergence speed and global searching ability of BFOA, they modify the swarming step and reproduction step, thereby improving the robustness of bacterial foraging (BF) and achieved fast convergence. Same authors employed few modifications to $\mathrm{BF}$ for threshold of brain magnetic resonance images by adaptively varying the step size of bacteria instead of fixed step size followed by ordinary bacterial foraging (Sathya and Kayalvizhi, 2011).

\section{RELATED WORK IN IMAGE COMPRES- SION}

Image compression is achieved by appropriate image thresholding and these thresholds are obtained with a principal of moment preserving and was proposed by Chen and Wen (1998) (Chen-Kuei and Wen-Hsiang, 2015). The proposed method achieved a high compression ratio with better reconstructed image quality. An image compression method which consumes less time and follows a strategy where thresholds are optimized with optimization techniques for which objective function is distortion (Kaur et al., 2007). Birge-Massart thresholding is inbuilt thresholding technique which is used for image compression and obtained results are compared with the uni-modal thresholding in terms of reconstructed image quality and compression ratio (Siraj, 2015). In (Tahere and Mohammad, 2009) Electrocardiography (ECG) signals are compressed by transforming the signal with the help of discrete wavelet transform. Another kind of image compression where image to be compressed is transformed to frequency domain with the help of bandlet and required bandlet coefficients are obtained with type II Fuzzy thresholding and results are compared with the ordinary thresholding (Rajeswari, 2012). Prashant and Ioana proposed a non-uniform thresholding and observed the effects of thresholding on reconstructed image quality (Prashant and Ioana, 2003). Tony and Zhou proposed a technique for noise removal and image compression in wavelet domain thresholding which is based on Partial Differential Equation (PDE) and it takes the advantage of variations in framework (Tony and Zhou, 2007). Image compression can also be performed with Multistage Lattice Vector Quantization (MLVQ) and by thresholding of Discrete Wavelet Transform (DWT) coefficients. Proposed combination tries to minimize the quantization error and its computational complexity is less compared to ordinary
VQ (Salleh and Soraghan, 2007). Kaveh et al., proposed a 2-D discrete wavelet transform based image thresholding by optimal thresholding the DWT coefficients with Particle Swarm Optimization (PSO) for image compression. They did three level decomposition of DWT and $62.5 \%$ of thresholds are assigned and optimized for the approximation coefficients and the remaining $37.5 \%$ equally assigned to horizontal, vertical, and diagonal coefficients (Kaveh et al., 2015). They compared the results with the Set Partition in Hierarchical Tree (SPHIT), Chrysafis, JPEG and JPEG-2000 and proved better in Peak Signal to Noise Ratio (PSNR) and Bits per Pixel (BPP).

In this paper, HBFOA-PSO based brain MRI image thresholding is proposed for image compression by optimizing the Renyi's entropy and Kapur's entropy for the first time and obtained results are compared with other optimization techniques such as BFOA, PSO, Moth-flame Optimization (MFO) Algorithm and GWO. Compressed image is further coded with runlength coding followed by arithmetic coding. Objective function value, standard deviation, Structural Similarity Index Measure (SSIM), PSNR, Weighted Peak Signal to Noise Ratio (WPSNR) and computational complexity are considered for the performance evaluation of proposed HBFOA-PSO based image thresholding. In all parameters the proposed algorithm performance is better as compared to other BFOA, PSO, MFO and GWO.

\section{PROBLEM FORMULATION OF OPTIMUM THRESHOLDING METHODS}

Initially all the required thresholds are selected randomly and these thresholds are optimized with the help of optimization techniques. In this paper, the objective function to be maximized with the optimization techniques is entropy techniques. The entropy techniques which are used in this paper are Renyi's entropy and Kapur's entropy. After successful optimization of thresholds, image is partitioned into object and background. Assume gray scale image which contains $L$ gray levels with range between 0 to $L-1(0,1,2, \ldots,(L-1))$. Then probability occurrence of pixel $P_{i}=h(i) / N(0<i<(L-1))$, where $h(i)$ is number of pixels corresponding to gray-level $L$ and $N$ is total number of pixels which is equal to $\sum_{i=0}^{L-1} h(i)$.

\section{CONCEPT OF RENYI'S ENTROPY}

Renyi's entropy is proposed by Sahoo in 1997 and it uses two probability distribution functions (pdf) one for object and another for background for thresholding of a gray level image (image contains 0 to 255 levels) 
(Prasanna et al., 1997). Let $\mathrm{G}$ is the gray levels of image and the range of these levels are $\{1,2, \ldots G\}$. Let $p_{i}=p_{1}$, $\mathrm{p}_{2} \ldots \mathrm{p}_{\mathrm{G}}$ is the probability distribution function (pdf) of corresponding gray levels. Among available pdfs some represents background (class A) and remaining represents object (class B). The probability distributions of the foreground and background classes are given in (Yudong and Lenan, 2011).

$$
\begin{aligned}
& p_{A}=\frac{p_{1}}{p^{A}}, \frac{p_{2}}{p^{A}}, \ldots \ldots \frac{p_{t}}{p^{A}} \\
& p_{B}=\frac{p_{t+1}}{p^{B}}, \frac{p_{t+2}}{p^{B}}, \ldots \ldots \frac{p_{G}}{p^{B}}
\end{aligned}
$$

Where $p^{A}=\sum_{i=1}^{t} p_{i} \quad p^{B}=\sum_{i=t+1}^{G} p_{i} \quad$ and

$$
p^{A}+p^{B}=1
$$

Renyi's entropy of a grayscale image with order $\alpha$ is given as

$$
H_{T}^{\alpha}=\frac{1}{1-\alpha} \ln \sum_{k=0}^{255}\left(p_{k}\right)^{\alpha}
$$

Where parameter $\alpha$ is a real positive constant. Form above equation when $\alpha \rightarrow 1$, Renyi's entropy $H_{T}^{\alpha}$ becomes Shannon entropy $\mathrm{H}_{\mathrm{T}}$ i.e $\lim _{\alpha \rightarrow 1} H_{T}^{\alpha}=H_{T}$

The Renyi's entropy for background and object is given in Eq. (3) and Eq. (4) respectively

$$
\begin{aligned}
& H_{p^{A}}^{\alpha}(t)=\frac{1}{1-\alpha} \ln \sum_{i=0}^{t}\left(\frac{p_{i}}{p^{A}}\right)^{\alpha} \\
& H_{p^{B}}^{\alpha}(t)=\frac{1}{1-\alpha} \ln \sum_{i=t+1}^{255}\left(\frac{p_{i}}{p^{B}}\right)^{\alpha}
\end{aligned}
$$

Let $H_{p^{A}}^{\alpha}(t)+H_{p^{B}}^{\alpha}(t)$ is maximum at $\mathrm{t}^{*}(\alpha)$ then

$$
t^{*}(\alpha)=\operatorname{Arg} \max _{t \in G}\left\{H_{p^{A}}^{\alpha}(t)+H_{p^{B}}^{\alpha}(t)\right\}
$$

From Eq. 5 it is observed that $t^{*}(\alpha)$ depends upon $\alpha$. From computer simulations

$$
t^{*}(\alpha)=\left\{\begin{array}{lc}
t_{1}^{*} & \text { if } 0<\alpha<1 \\
t_{2}^{*} & \text { if } \alpha \rightarrow 1 \\
t_{3}^{*} & \text { if } 1<\alpha<\infty
\end{array}\right.
$$

Where $t_{1}^{*}, t_{2}^{*}$ and $t_{3}^{*}$ are different gray values. When $\alpha$ move towards 1 , then the optimal threshold $t_{2}^{*}$ obtained with Renyi's entropy is equal to threshold obtained with the maximum entropy sum method and

when $\alpha>1$, optimal threshold $t_{3}^{*}$ with Renyi's entropy is equal to threshold with the entropic correlation method. The optimal threshold value of the Renyi's entropy is calculated by the following formula with $t_{1}^{*}, t_{2}^{*}$ and $t_{3}^{*}$.

$t_{c}^{*}=t_{[1]}\left[p\left(t_{[1]}\right)+\frac{1}{4} w \beta_{1}\right]+\frac{1}{4} t_{[2]} w \beta_{2}+t_{[3]}\left[1-p\left(t_{[3]}\right)+\frac{1}{4} w \beta_{3}\right]$

Where $t_{[1]}, t_{[2]}$ and $t_{[3]}$ are order statistics of the gray values $t_{1}^{*}, t_{2}^{*}$ and $t_{3}^{*}$,

$$
\begin{aligned}
p(t) & =\sum_{i=1}^{t} p_{i}, w=p\left(t_{[3]}\right)-p\left(t_{[1]}\right), \text { and } \\
\left(\beta_{1}, \beta_{2}, \beta_{3}\right) & = \begin{cases}(1,2,1) & \text { if }\left|t_{[1]}-t_{[2]}\right| \leq 5 \text { and }\left|t_{[2]}-t_{[3]}\right| \leq 5, \\
(1,2,1) & \text { if }\left|t_{[1]}-t_{[2]}\right|>5 \text { and }\left|t_{[2]}-t_{[3]}\right|>5, \\
(0,1,3) & \text { if }\left|t_{[1]}-t_{[2]}\right| \leq 5 \text { and }\left|t_{[2]}-t_{[3]}\right|>5, \\
(3,1,0) & \text { if }\left|t_{[1]}-t_{[2]}\right|>5 \text { and }\left|t_{[2]}-t_{[3]}\right| \leq 5 .\end{cases}
\end{aligned}
$$

The optimal threshold value $t_{c}^{*}$ can be viewed as an image dependent weighted average of $t_{1}^{*}, t_{2}^{*}$ and $t_{3}^{*}$ and thus $\min \left\{t_{1}^{*}, t_{2}^{*}, t_{3}^{*}\right\} \leq t_{c}^{*} \leq \max \left\{t_{1}^{*}, t_{2}^{*}, t_{3}^{*}\right\}$ that is, $t_{[1]} \leq t_{c}^{*} \leq t_{[3]}$. This shows that the maximum entropy sum method or the entropic correlation method does not succeed in providing a good threshold value for a gray scale image but the Renyi's entropy provides a better threshold value.

\section{CONCEPT OF KAPUR'S ENTROPY}

Kapur developed an algorithm for bi-level thresholding which is as follows: The objective function is

$$
J(t)=H_{0}+H_{1}
$$

$$
\text { Where } \begin{aligned}
H_{0} & =-\sum_{i=0}^{t-1} \frac{p_{i}}{w_{0}} \ln \frac{p_{i}}{w_{0}}, & w_{0} & =\sum_{i=0}^{t-1} p_{i} \\
H_{1} & =-\sum_{i=t}^{L-1} \frac{p_{i}}{w_{1}} \ln \frac{p_{i}}{w_{1}}, & w_{1} & =\sum_{i=t}^{L-1} p_{i}
\end{aligned}
$$

When objective function Eq. (9) is maximum then thresholds are optimal threshold. For multi-level thresholding Eq. (9) becomes

$$
J\left(t_{0}, t_{1}, \ldots \ldots . t_{m}\right)=H_{0}+H_{1}+H_{2}+\ldots \ldots+H_{m}
$$

Where $\mathrm{m}$ is number of thresholds to be optimized and 


$$
\begin{array}{ll}
H_{0}=-\sum_{i=0}^{t_{1}-1} \frac{p_{i}}{w_{0}} \ln \frac{p_{i}}{w_{0}}, & w_{0}=\sum_{i=0}^{t_{1}-1} p_{i} \\
H_{1}=-\sum_{i=t_{1}}^{t_{2}-1} \frac{p_{i}}{w_{1}} \ln \frac{p_{i}}{w_{1}}, & w_{1}=\sum_{i=t_{1}}^{t_{2}-1} p_{i} \\
H_{2}=-\sum_{i=t_{2}}^{t_{3}-1} \frac{p_{i}}{w_{2}} \ln \frac{p_{i}}{w_{2}}, & w_{2}=\sum_{i=t_{2}}^{t_{3}-1} p_{i} \\
H_{m}=-\sum_{i=t_{m}}^{L-1} \frac{p_{i}}{w_{m}} \ln \frac{p_{i}}{w_{m}}, & w_{m}=\sum_{i=t_{m}}^{L-1} p_{i}
\end{array}
$$

Image compression with the Renyi's entropy and Kapur entropy with two level thresholding proved efficient, but when threshold levels are increasing (multilevel thresholding) Renyi's entropy and Kapur's entropy takes much time for simulation and time increases exponential with levels. To improve the performance of Renyi's entropy and Kapur's entropy and to reduce the simulation time, few applications of soft computing techniques such as BFOA, PSO, MFO, and GWO for image thresholding are proposed, hence effective image compression. These techniques are to maximize the Renyi's entropy and Kapur entropy as given in Eq. (5) and Eq. (9).

\section{PROPOSED HBFOA-PSO}

The main objective of this paper is to get optimal thresholds which leads to a better reconstructed image quality at high compression ratio. The optimal thresholds are obtained by cascaded combination of bacterial foraging optimization algorithm and particle swam optimization. The bacterial foraging optimization algorithm is global search algorithm but it may be trapped into local optimal problem and delay in execution time (convergence time) because of random chemotaxis steps in the procedure of algorithm and to get global solution, a theory of swarming is commenced in the structure of HBFOA-PSO. In order to find the advantage of proposed HBFOA-PSO approach, the results are compared with individual BFOA and PSO approaches. Each of algorithms is explained below.

\section{OVERVIEW OF BACTERIA FORAGING OPTIMIZATION ALGORITHM}

In 2002, a new optimization technique is proposed by Passino which is based on the foraging behavior of bacteria called Bacteria Foraging Optimization Technique (BFOA) (Passino, 2002). E.Coli bacteria always searches for nutrients to enhance the energy levels per unit time. Some of the bacteria search for nutrients by communicating with each other. In general, bacteria search for nutrients with the help of tumbling or swarming and chemotaxis step. The BFOA performance is better than other optimization techniques because of its advanced algorithm structure. In BFOA step of walk follows a Gaussian distribution function in searching food instead of Levy flight which is used in cuckoo search algorithm.

In this paper the objective function which is optimized with BFOA is entropy (Renyi's or Kapur's entropy). The bacteria moves in such a way that in each iteration the objective function is maximum. Because of this, each bacterium carries different objective function value in each iteration. Among all the objective function values of bacteria, the highest value is carried to next iteration. The remaining bacteria always try to move towards the highest objective function value bacteria and attain further highest values after successful final iteration. In this way all the bacteria attain global optimal solution. The BFOA attain this optimal solution with four cascaded steps: 1. Chemotaxis, 2. Swarming, 3. Reproduction and 4. Elimination-dispersal. The four steps in BFOA are explained below.

1. Chemotaxis: Chemotaxis step is a crucial step in BFOA while searching for food and it illustrates intelligence applied by the bacteria while searching for food. The bacteria try to move towards the better solution by taking either thumbling or swimming. In BFOA, each bacteria move to its better position by taking 8-neighbourhood positions derivative. After derivative, it finds which bacteria has maximum objective function and remaining bacteria follow the maximum objective function bacteria. The steps of Chemotaxis are as follow:

Tumbling: In this step bacteria moves randomly in a particular direction where high nutrients are available in the search space. Initially all the bacteria are having natural nutrients. This process is known as tumbling and is shown in Fig. 1.

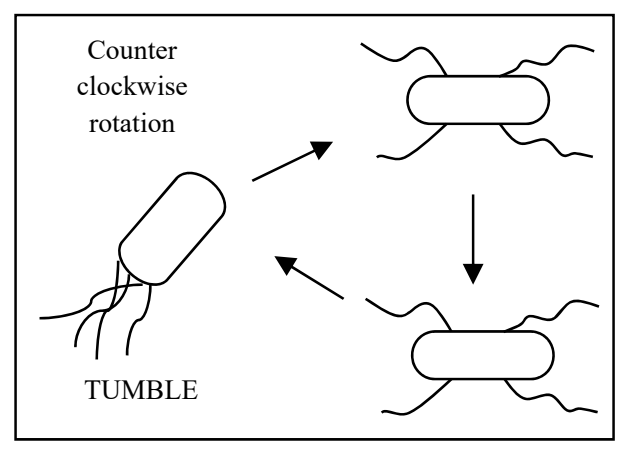

Fig. 1. Tumbling of bacterium

Swimming up: After successful and sufficient nutrient from tumbling, the bacteria move in the same direction if nutrients are further increasing or else it take 
swimming step. This swimming movement is called swimming up.

Swimming down: If the direction of movement decrease the bacteria nutrients then movement is called swimming down. When bacteria experience swimming down then immediately it changes its direction. This process is shown in Fig. 2.

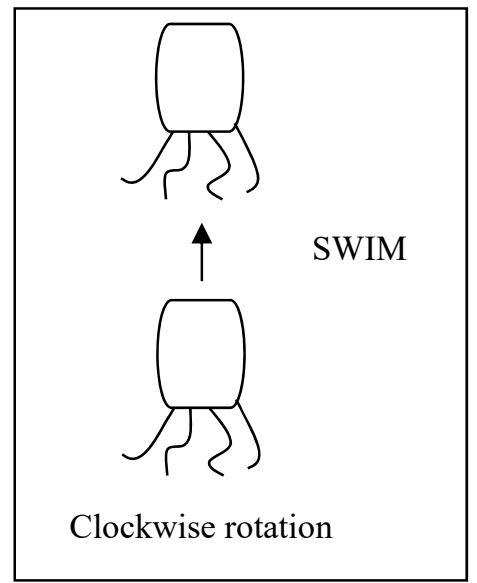

Fig. 2. Bacterial swim

The chemotactic step of bacteria is mentioned in Eq. (11).

$$
\theta^{i}(j+1, k, l)=\theta^{i}(j, k, l)+C(i) \cdot \frac{\Delta(i)}{\sqrt{\Delta^{i}(i) \Delta(i)}}
$$

Where $C$ (i) is step size and $\Delta$ (i) is the random number lying between $[0,1]$.

2. Swarming: The collection of bacteria which are at higher nutrients will send information through signal to other bacteria. So rest of the bacteria will try to move towards the higher nutrients direction and avoids the direction of movement towards the lower nutrients. This step of process is called swarming and is described in below equation.

$$
\begin{aligned}
& J_{c c}\left(\theta,(P(j, k, l))=\sum_{i=1}^{S} J_{c c}\left(\theta, \theta^{i}(P(j, k, l))=\right.\right. \\
& \sum_{i=1}^{S}\left[-d_{\text {attra }} \exp \left(-W_{\text {attra }} \sum_{m=1}^{P}\left(\theta_{m}-\theta_{m}^{i}\right)^{2}\right)\right]+ \\
& \sum_{i=1}^{S}\left[h_{\text {repel }} \exp \left(-W_{\text {repel }} \sum_{m=1}^{P}\left(\theta_{m}-\theta_{m}^{i}\right)^{2}\right)\right]
\end{aligned}
$$

3. Reproduction: All the bacteria fitness values are shortlisted ascending or descending based on maximization problem or minimization problem respectively. In this paper, the objective function is to be maximized so, all the bacteria are arranged in ascending order based on their fitness values or objective function value. In this step the bacteria with lowest nutrients would die. In general, around half of bacteria die in this step and new bacteria is generated by asexual between two highest nutrients bacteria. Indirectly in this step among the available bacteria, half of bacteria die and these are replaced with newly generated bacteria to always maintain constant bacteria in search space. The process of new bacteria generation is called conjugation.

4. Elimination-dispersal: In some cases bacteria may experience a sudden change in environmental conditions like hike in temperature or in humidity. Then bacteria undergoes third step i.e. reproduction where bacteria may die because of sudden changes and new bacteria are generated by a sexual relation between two bacteria. Some bacteria may move to the nearest safest place.

BFOA algorithm:

$$
\begin{aligned}
& \text { For } \mathrm{l}=1: \mathrm{Ned} \\
& \text { For } \mathrm{k}=1: \mathrm{Nre} \\
& \qquad \begin{array}{l}
\text { For } \mathrm{j}=1: \mathrm{Nc} \\
\text { For } \mathrm{i}=1: \mathrm{S} \\
\mathrm{J}(\mathrm{i}, \mathrm{j}, \mathrm{k}, \mathrm{l})=\mathrm{J}(\mathrm{i}, \mathrm{j}, \mathrm{k}, 1)+\mathrm{Jcc}\left[\theta^{i}(j, k, l), P(j, k, l)\right] \\
\quad \Delta_{m}(i), m=1,2, \ldots \ldots \ldots, p \\
\theta^{i}(j+1, k, l)=\theta^{i}(j, k, l)+C(i) \cdot \frac{\Delta(i)}{\sqrt{\Delta^{i}(i) \Delta(i)}}
\end{array}
\end{aligned}
$$

Calculate $\mathrm{J}(\mathrm{i}, \mathrm{j}+1, \mathrm{k}, \mathrm{l})$

$\mathrm{m}=0$

While $\mathrm{m}<\mathrm{Ns}$

$\mathrm{m}=\mathrm{m}+1$

If $J(\mathrm{i}, \mathrm{j}, \mathrm{k}, \mathrm{l})>J_{\text {last }}$

Update $J_{\text {last }}$

$\theta^{i}(j+1, k, l)=\theta^{i}(j, k, l)+C(i) \cdot \frac{\Delta(i)}{\sqrt{\Delta^{i}(i) \Delta(i)}}$

else $m=N s$

End if

End while

End i-for

End j-for

End k-for

For $\mathrm{i}=1: \mathrm{S}$

$J_{\text {health }}^{i}=\sum_{j=1}^{N c+1} J(i, j, k, l)$

End i-for

End $\mathrm{k}$-for

For $\mathrm{i}=1: \mathrm{S}$ 
If rand ()$<$ Ped

Eliminate bacterium and initialize randomly its replacement

End if

End i-for

End 1-for

End BFOA

\section{OVERVIEW OF PARTICLE SWARM OPTIMIZATION}

PSO is proposed by Kennedy and Eberhart in the year 1995 and it is a stochastic approach under swarm intelligence that mimics how the particles are flying to get the best food location (Kennedy and Eberhart, 1995). Each individual particle adaptively updates their velocity and position within the search depends on the previous experience of its own search and the experiences of other particles in the population. Each particle is assigned with a memory by which it can store the best food location it ever visited during its journey. Its best food location is named as Pbest and the best food location of the group taken as one is stored as Gbest. The initial positions for Pbest and Gbest are different. It is proved to give the best results in obtaining the global minima or maxima. However, obtaining the global minima about the optimum value is a challenging issue, whenever multiple minima exist. This algorithm does not involve cross-over or mutation operators. It only depends on the initialization of the control parameters, the size of the swarm, the objective function and the maximum number of iteration. It does not depend on the initial conditions and the gradient values.

The advantages of using PSO are computationally less expensive, much simple to implement, Less CPU time and memory requirement. The modified velocity of each particle is given by

$$
\begin{aligned}
& v(t+1)=v(t)+c_{1} r_{1}(\text { Pbest }-x(t))+ \\
& c_{2} r_{2}(\text { Gbest }-x(t))
\end{aligned}
$$

The modified position of each particle is given by

$$
x(t+1)=x(t)+v(t+1)
$$

PSO Algorithm:

Step 1: Initialization of each individual particle in the population with random position and random velocity.
Step 2: Calculate the objective function of each particle with Eq. (5) and Eq. (9). If the current cost is higher than the best value so far calculated, then it is stored in Pbest.

Step 3: Choose the particle with the highest objective function value of all particles. The position of this particle is Gbest.

Step 4: Calculate the new velocity and position of each particle according to the Eq. (13) and Eq. (14).

Step 5: Repeat the above steps from 2-4 until maximum iterations or maximum criteria is not attained.

\section{HBFOA-PSO ALGORITHM}

The HBFOA-PSO algorithm combines BFOA and PSO algorithms, so it takes the advantages and disadvantages of both techniques. The aim is to share information between PSO and BFOA that leads to generation of healthy bacteria by dispersal and elimination. The major drawback with the BFOA is, step of tumbling is random so achieving a global solution is difficult. Where as in the proposed hybrid BFOA-PSO, the step of tumbling is not random and these tumbling steps are optimized with the PSO. The global best solution or better suitable tumbling step from the PSO is given as input to BFOA. Tumbling step is updated when BFOA is in first step. The parameters required for hybrid BFOA-PSO are given below.

Step 1: Initialization of parameters for both BFOA and PSO:

$\mathrm{p}=$ Dimensions of the problem;

$\mathrm{S}=$ population size or number of particle in case of $\mathrm{PSO}$ and number of bacteria in case of BFOA;

Ns = swimming length after tumbling operation when bacteria is in chemotaxis loop;

$\mathrm{Nc}=$ stopping criteria or maximum iterations of the algorithm;

Nre $=$ Maximum number of reproduction steps;

Ned = Maximum number of steps in elimination and dispersal loop;

Ped $=$ the probability of elimination and dispersal;

$\mathrm{C}(\mathrm{i})=$ step of walk in tumbling stage and is random in BFOA algorithm;

$$
d_{a t}, w_{a t}, h_{r e}, w_{r e}=\text { Bacteria attractive and }
$$
repellent coefficients;

$\Delta(\mathrm{p}, \mathrm{i})=$ Bacteria direction in current iteration;

$P(i, j)=$ Bacteria position in current iteration; 
$c_{1}, c_{2}=$ PSO cognitive constant and social acceleration coefficient respectively;

$\mathrm{r} 1, \mathrm{r} 2=$ Random numbers between 0 to 1 in case of

PSO;

Step 2: Elimination and dispersal loop: $1=1+1$.

Step 3: Reproduction loop: $\mathrm{k}=\mathrm{k}+1$.

Step 4: Chemotaxis loop: $\mathrm{j}=\mathrm{j}+1$.

Substep a: For $\mathrm{i}=1,2, \ldots, \mathrm{S}, \mathrm{i}^{\text {th }}$ bacteria move with step which is as follows

Calculate all bacteria objective value, $\mathrm{J}(\mathrm{i}, \mathrm{j}, \mathrm{k}, \mathrm{l})$;

Then new fitness function

$\left.\mathrm{J}(\mathrm{i}, \mathrm{j}, \mathrm{k}, \mathrm{l})=\mathrm{J}(\mathrm{i}, \mathrm{j}, \mathrm{k}, \mathrm{l})+\mathrm{J}_{\mathrm{cc}}\left(\theta^{i}(j, k, l), P(j, k, l)\right]\right) ;$

Assign J_last $=\mathrm{J}(\mathrm{i}, \mathrm{j}, \mathrm{k}, \mathrm{l})$.

Substep b: For $\mathrm{i}=1,2, \ldots, \mathrm{S}$ bacteria decided to take either tumbling or swimming $\Delta(i)$, that is randomly generated number between 0 to 1 in first iteration for all the bacteria or for all populations. From second iteration onwards tumbling position and direction are optimized with PSO. Bacteria moves towards better direction with $\theta^{i}(j+1, k, l)=\theta^{i}(j, k, l)+C(i) \cdot \frac{\Delta(i)}{\sqrt{\Delta^{i}(i) \Delta(i)}}$

Where $\mathrm{j}, \mathrm{k}$ and $\mathrm{l}$ are index of Chemotaxis step, reproduction \& Elimination and dispersal respectively.

Which leads $i^{\text {th }}$ bacteria will move with a step size C(i) in tumbling stage

Calculate $\mathrm{J}(\mathrm{i}, \mathrm{j}, \mathrm{k}, \mathrm{l})=\mathrm{J}(\mathrm{i}, \mathrm{j}, \mathrm{k}, \mathrm{l})+$

$\operatorname{Jcc}\left[\theta^{i}(j, k, l), P(j, k, l)\right]$

Swimming stage

i. Assume $\mathrm{m}=0$ (counter for swim length).

ii. While $\mathrm{m}<\mathrm{Ns}$

Let $m=m+1$

$$
\text { If } J(\mathrm{i}, \mathrm{j}+1, \mathrm{k}, \mathrm{l})>J_{\text {last }} \text {, let } J_{\text {last }}=|J(\mathrm{i}, \mathrm{j}+1, \mathrm{k}, \mathrm{l})|
$$$$
\text { and } \theta^{i}(j+1, k, l)=\theta^{i}(j, k, l)+C(i) \cdot \frac{\Delta(i)}{\sqrt{\Delta^{i}(i) \Delta(i)}}
$$

Use this new

$\theta^{i}(\mathrm{j}+1, \mathrm{k}, \mathrm{l})$ to compute new $\mathrm{J}(\mathrm{i}, \mathrm{j}+1, \mathrm{k}, \mathrm{l})$.

iii. Else, let $\mathrm{m}=$ Ns. This is the end of the while statement for swim.

Substep c: Go to next bacterium $(i+1)$ if $i \neq S$ (i.e., go to substep $b$ to process the next bacterium)

Step 5: Evaluate the local best position for each bacterium and global best position.
Step 6: Update the velocity and position of bacterium using PSO. Update the vector $\Delta(\mathrm{p}, \mathrm{i})$.

Step 7: If $\mathrm{j}<\mathrm{Nc}$, go to step 4 and continue chemotaxis until life of the bacteria is over.

Step 8: Reproduction:

Substep a: For the given $\mathrm{k}$ and 1 , and for each $\mathrm{i}=1$, 2. . . S, find health of bacterium $\mathrm{i}, j_{H L}^{i}$ as $j_{H L}^{i}=$ $\sum_{j=1}^{N_{c}+1} j(i, j, k, l) \cdot j_{H L}^{i}$ gives a measure of how many nutrients it got over its lifetime and how successful it was at avoiding noxious substances. Sort bacteria and chemotactic parameters $\mathrm{C}$ (i) in order of ascending $j_{H L}$ value (Lower $j_{H L}$ value means lower health).

Substep b: The $\mathrm{Sr}=\mathrm{S} / 2$, bacteria with the lowest $j_{H L}$ values are removed and other $\mathrm{Sr}$ bacteria with the best $j_{H L}$ value split. New bacteria that are made are placed at the same location as their parent.

Step 9: If $\mathrm{k}<$ Nre, go to step 3.

Step 10: Elimination and dispersal: For $\mathrm{i}=1,2 \ldots \mathrm{S}$ with probability $P_{e d}$, elïminate and disperse each bacterium.
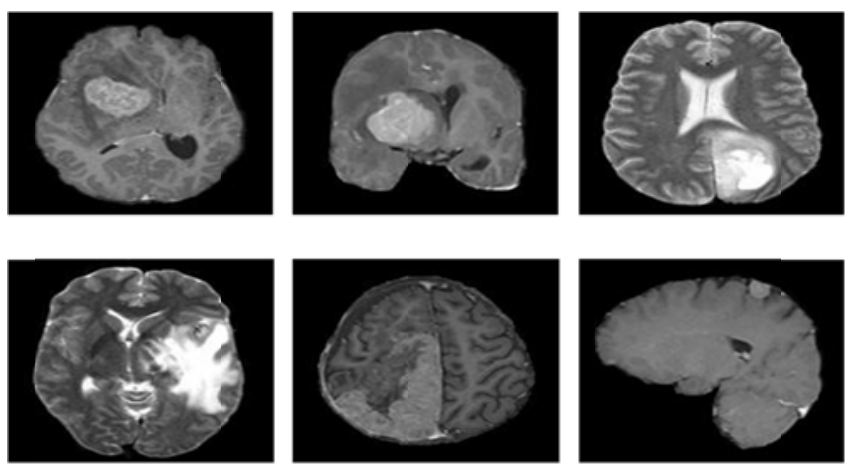

Fig. 3: Test images (clockwise) a) Astrocytoma b) Coronary T1 Astrocytoma c) Glioma d) Metastatic e) PNET f) Meningioma

\section{RESULTS}

For evaluation of experiments, six still images of size 256x256; namely "Astrocytoma", "Coronary T1 Astrocytoma", "Glioma", "Metastatic", "PNET", and "Meningioma" with a 8" bits per pixel amplitude resolution are taken as shown in Fig. 3. These images are captured from Siemens-Area MRI scanner equipment, in which image is captured on slice of thickness $1 \mathrm{~mm} \times 1 \mathrm{~mm} \times 1 \mathrm{~mm}$ by 48 multi channels with 1.5 Tesla magnetic field intensity. The advanced technology used in this equipment is Magnetom Avanto-Tim technology. Experiments are conducted for diagnosis of brain tumour on four different patients with age $3,32,35$, and 42 . The program is written on 
Matlab15a software tool for execution of five algorithms. The number of solutions assigned initially is 100 and the maximum iteration number is assumed as 20.

\section{DISCUSSION}

In this paper, the performance of the proposed algorithm is explained in three sections: Evaluation of Algorithm performance, performance evaluation of proposed method in identifying tumour region and performance evaluation of proposed method for image compression.

\section{EVALUATION OF ALGORITHM PER- FORMANCE}

This section explains the performance of proposed method against other algorithms in terms of objective/maximum fitness function, mean value, standard deviation, and elapsed time or computational time.

FITNESS FUNCTION: It explains how best a solution is fit to the problem. In this paper, Kapur's entropy and Renyi's entropy are considered as fitness function for effective and efficient image thresholding. Here the number of thresholds are 5 and are optimized with the proposed HBFOA-PSO. Table 1 shows the fitness value obtained with the proposed HBFOA-PSO is larger when compared with the other algorithms.

MEAN AND STANDARD DEVIATION: All the optimization techniques are run more than 20 times and some randomness is involved in the execution process of the algorithm. Because of randomness, the algorithm never generates the same solution all times. The stability measuring parameter of the algorithm is mean and standard deviation. Mean value is the ratio of sum of maximum fitness value obtained with each run to total number of runs. Standard deviation is defined as a quantity expressing by how much the members of maximum fitness value differ from the mean value. From Table 1 it is clear that mean value and standard deviation of the proposed method is better than other algorithms.

\section{COMPUTATIONAL TIME}

It is measured in seconds and is total time taken by the algorithm to produce outcome or results. The proposed HBFOA-PSO algorithm computational time is little bit higher as compared with others because of cascading BFOA and PSO and is illustrated in Table 1. In comparison with the Renyi's entropy and Kapur's entropy, computational time of Renyi's entropy is a little higher.

\section{PERFORMANCE EVALUATION OF PROPOSED METHOD IN IDENTIFYING TUMOUR REGION}

As disused in introduction section, in this section first it is explained how the proposed HBFOA-PSO is better in identification of brain tumour area as compared with other optimization techniques with maximizing Renyi's entropy and Kapur's entropy. This section explains visual clarity of tumour regions with the proposed method and other algorithms. For testing of HBFOA-PSO six images of different tumours in brain are chosen and all the images are in .jpg format. Fig. 4 to Fig. 9 show the optimal threshold images with optimized thresholds and which partition the image into clusters/groups and from these clusters one can clearly distinguish the tumour area and edema area in tumour affected brain image.

Fig. 4 illustrates the threshold image of a female patient of age 42 and she is suffering with metastatic bronchogenic carcinoma brain tumour. From Fig. 4 it is observed that, visually tumour area and edema area are clearly partitioned with the proposed HBFOA-PSO compared with other algorithms. Input image shows that tumour is affected in left temporal region of the brain (high intensity region) but left hemisphere of the patient brain is totally inflamed as consequences of the allencompassing surgery. For surgery radiologist may confuse in identifying the exact tumour location and it is possible with the HBFOA-PSO.

Proposed HBFOA-PSO efficiency is tested on low grade Glioma brain tumour image and proved better compared to other algorithms and shown in Fig. 5. This Glioma brain tumour image is obtained from a patient of age 35 and tumour is identified in the left occipital area. Visual identification of tumour area and edema area are very clear with the proposed HBFOA-PSO.

Fig. 6 shows the threshold image of patient of age 35 and was suffering with meningioma tumour. Reasonable occurrence of calcified pathology is reported in the right parietal convexity with a dural tail. The proposed HBFOA-PSO clearly identifies area of tumour in meningioma tumour image and this demonstrates the toughness and competence of the proposed HBFOA-PSO in identifying the tumour area of demanding clinical data.

Fig. 7 and Fig. 8 shows the threshold image of patient of age 32 and was suffering with high grade Astrocytoma tumour and these figures demonstrate the 
efficiency and effectiveness of the proposed HBFOAPSO. The HBFOA-PSO visually shows the tumour area between right ganglio capsular region and thalamus and rest area. In spite of the effacement of ipsilateral ventricular system, which occurred due to the presence of intense edema, the suggested HBFOA-PSO has successfully identified the tumour region.

In general tumour becomes cancer and life time of the patient depends on the stage of tumour level and when this level is above four then lifetime of patient is around six months. Identifying the tumour at early stage is a difficult task because there is no much difference in intensity level as shown in Fig. 9. Even with this kind of images also proposed HBFOA-PSO shows better results as compared to other algorithms.

Primitive Neuro Ectodermal Tumour (PNET) is a massive tumour which is mostly appearing in the children at age of below 25 years and it is due to primitive or undeveloped cells in the brain and these cells try to extend to entire nervous system of the brain. They appear similar to meduloblastoma and were once considered a single tumour. PNET contain cysts and dead cells and around the tumour fluids are distributed unequally. As compared to medulloblastoma, PNET is massive aggressive tumour which mostly affects the cerebral hemispheres of the brain. Fig. 9 shows the patient brain image of age 3 and is obtained while doing post radio therapeutic diagnosis and was suffering with supratentorial tumour. The effectiveness of the proposed HBFOA-PSO algorithm is that it works well in identifying PNET tumour at early stage as compared to other state-of-art algorithms.

\section{Kapur's Entropy}

Input image

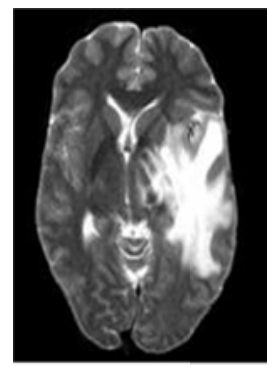

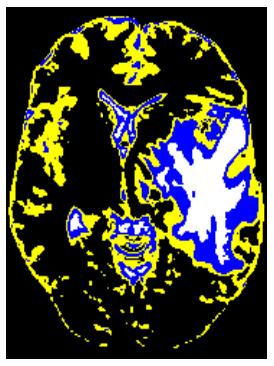

GWO

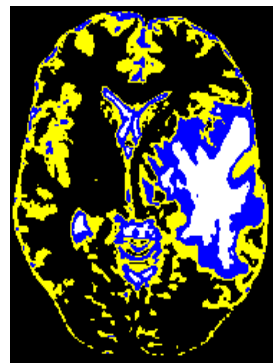

PSO

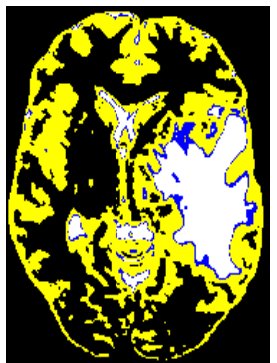

BFOA
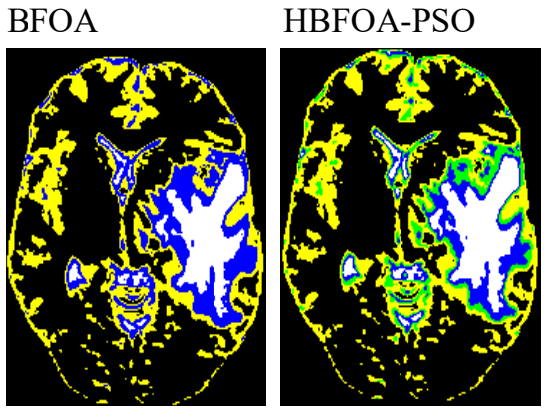

\section{Renyi's Entropy}
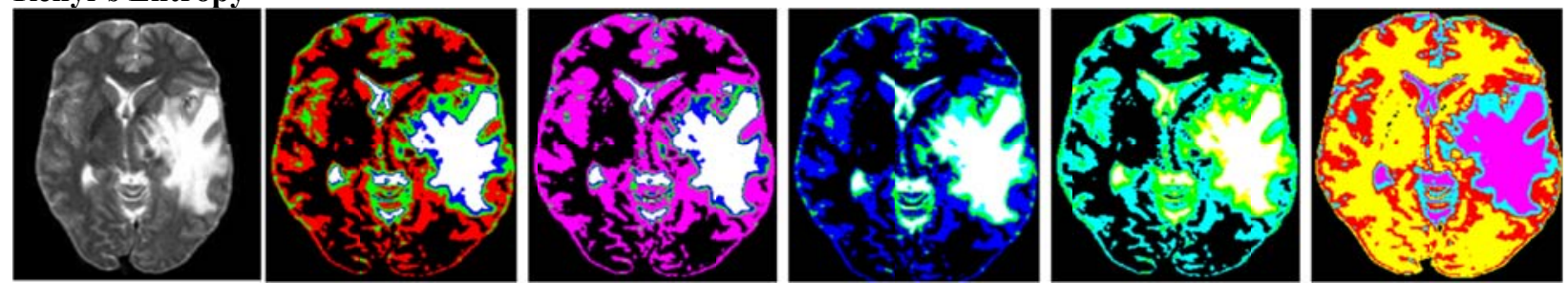

Fig. 4. Metastatic Bronchogenic Carcinoma affected brain image and optimal thresholld images obtained with five algorithms 


\section{Kapur's Entropy}
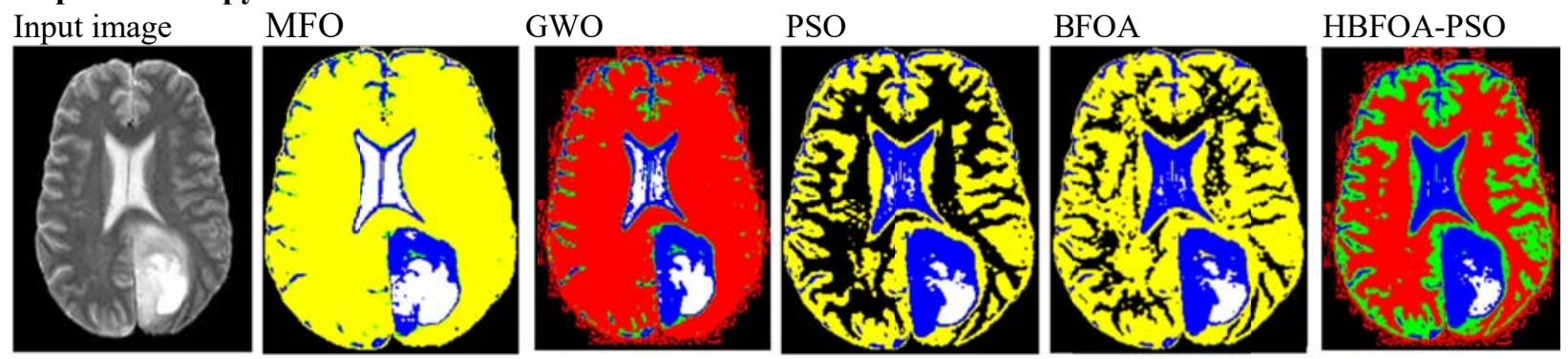

\section{Renyi's Entropy}
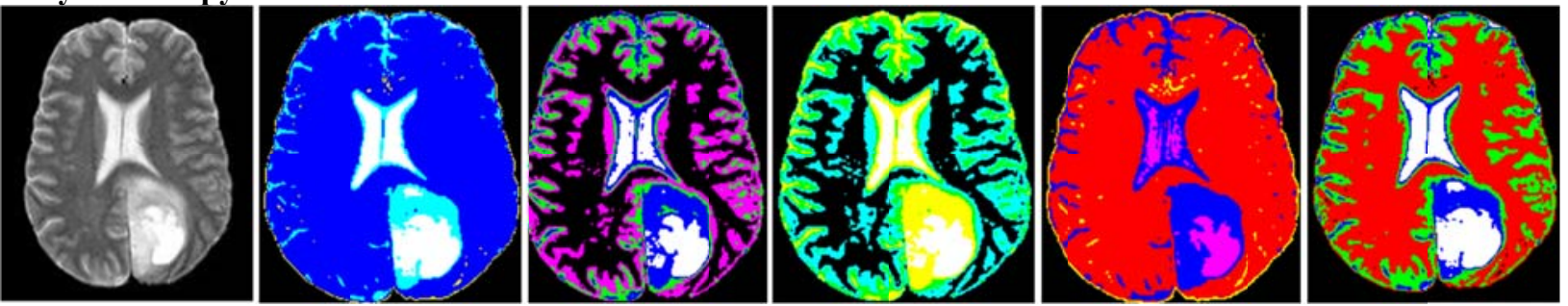

Fig. 5. Glioma affected brain image and optimal threshold images obtained with five algorithms

\section{Kapur's Entropy}

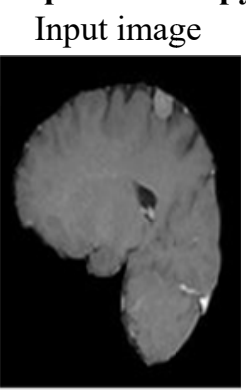

\begin{abstract}
MFO
\end{abstract}
GWO
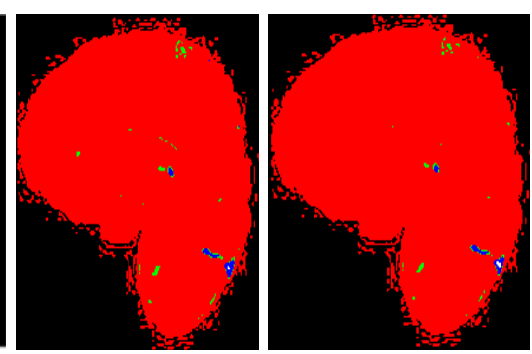

PSO

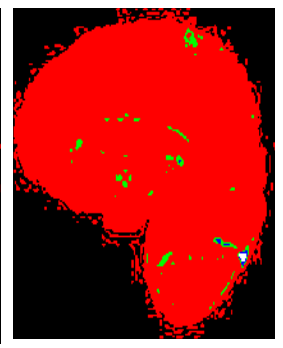

BFOA

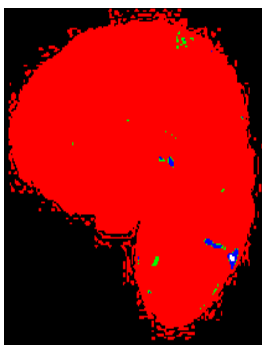

HBFOA-PSO

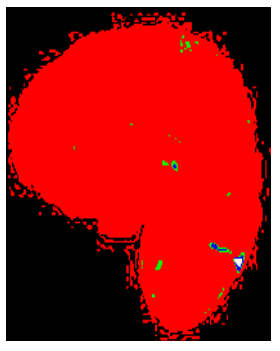

\section{Renyi's Entropy}
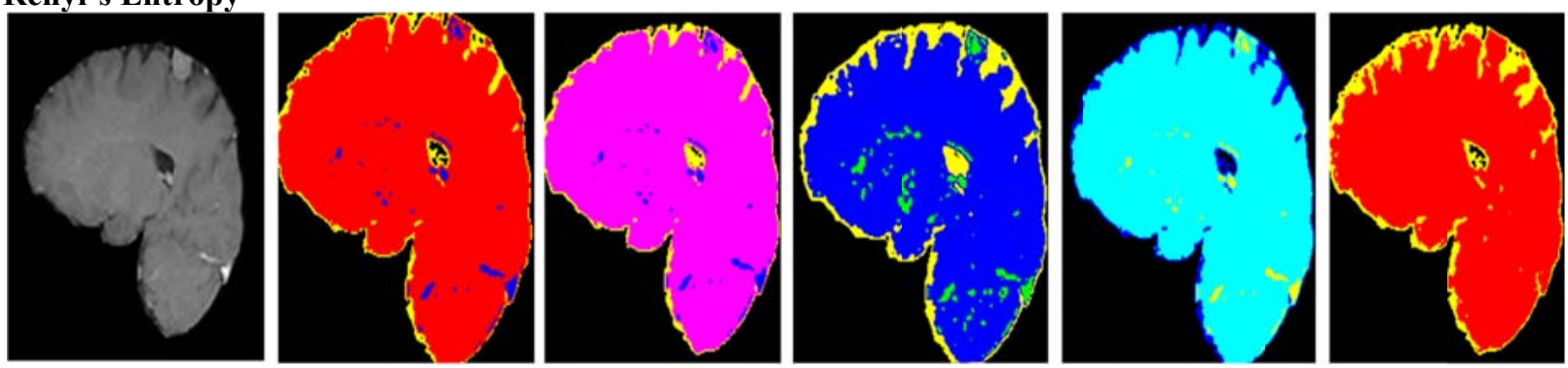

Fig. 6. Meningioma affected brain image and optimal threshold images obtained with five algorithms 


\section{Kapur's Entropy}

Input image
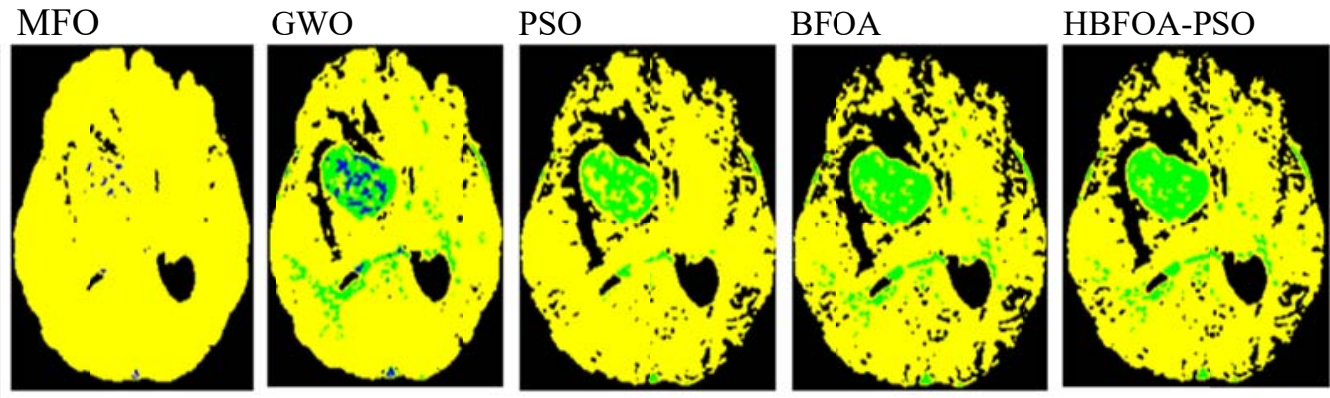

\section{Renyi'sEntropy}
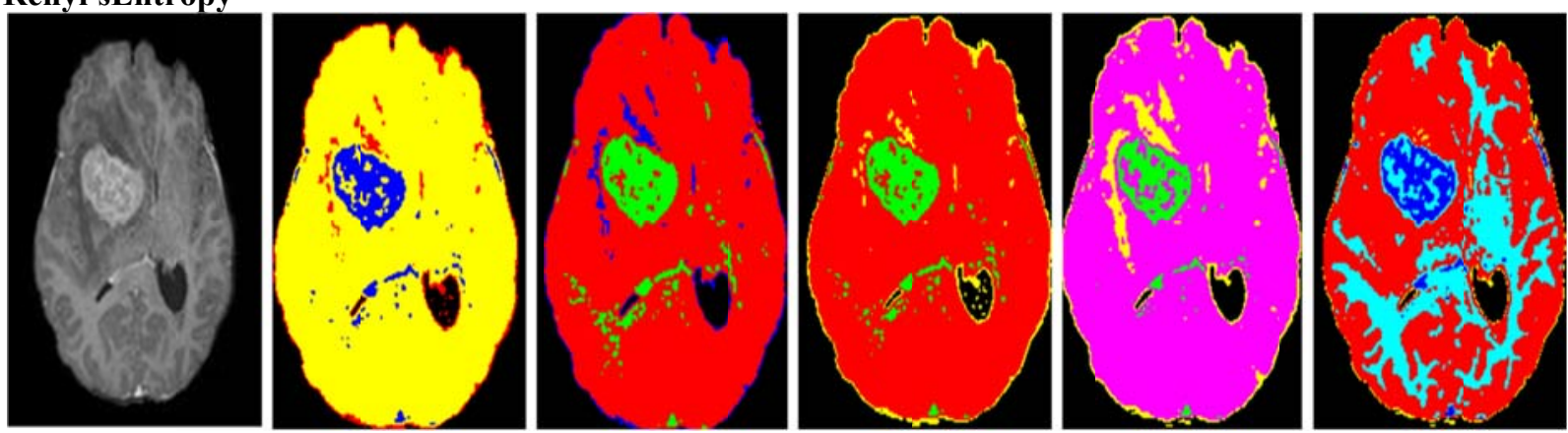

Fig. 7. Astrocytoma tumour affected brain image and optimal threshold imagesobtained with five algorithms

\section{Kapur's Entropy}
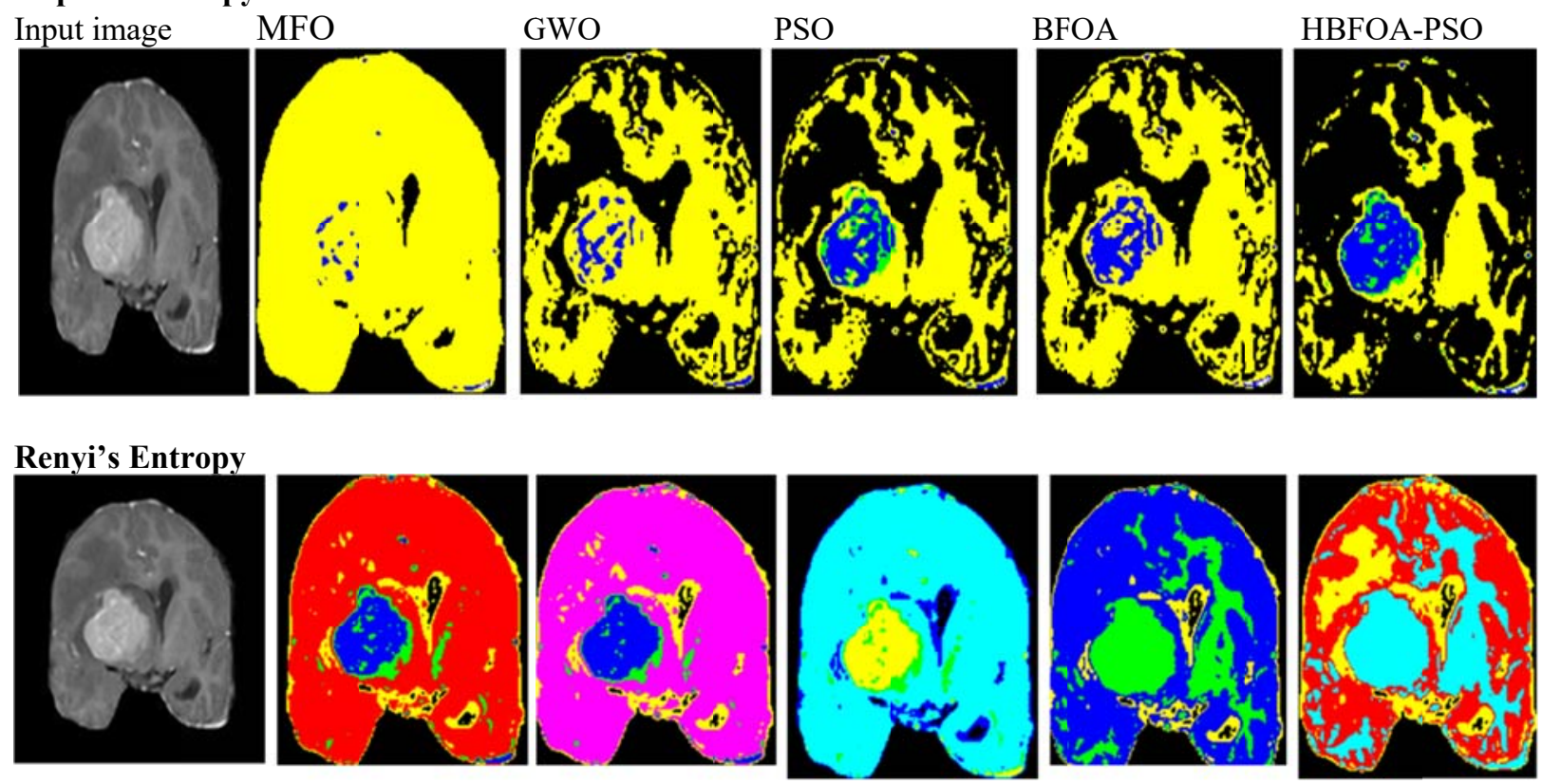

Fig. 8. Coronary T1 Astrocytoma tumour affected brain image and optimal threshold images obtained with five algorithms 


\section{Kapur's Entropy}
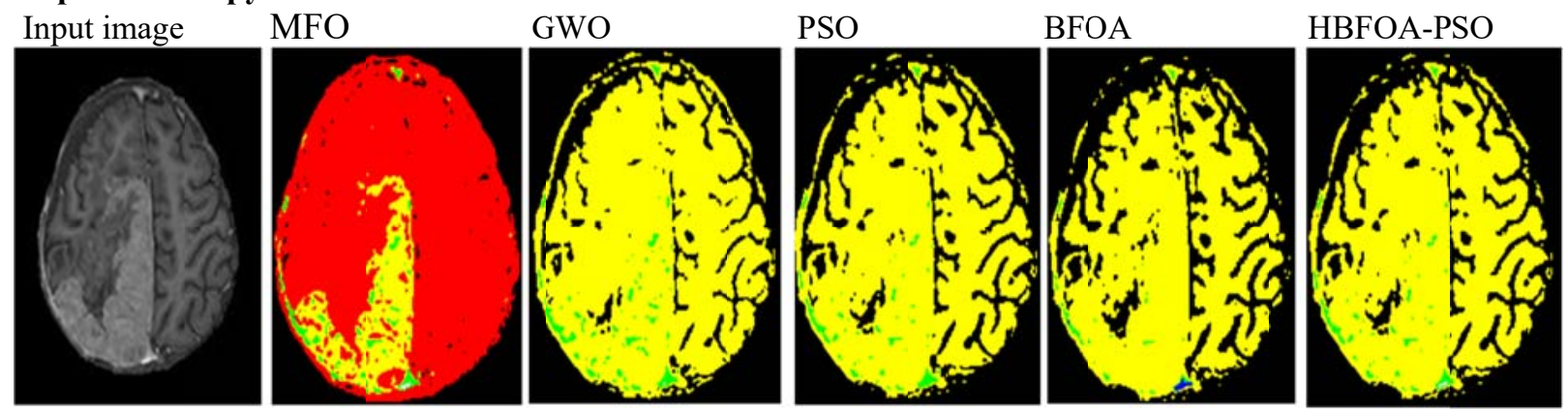

\section{Renyi's Entropy}
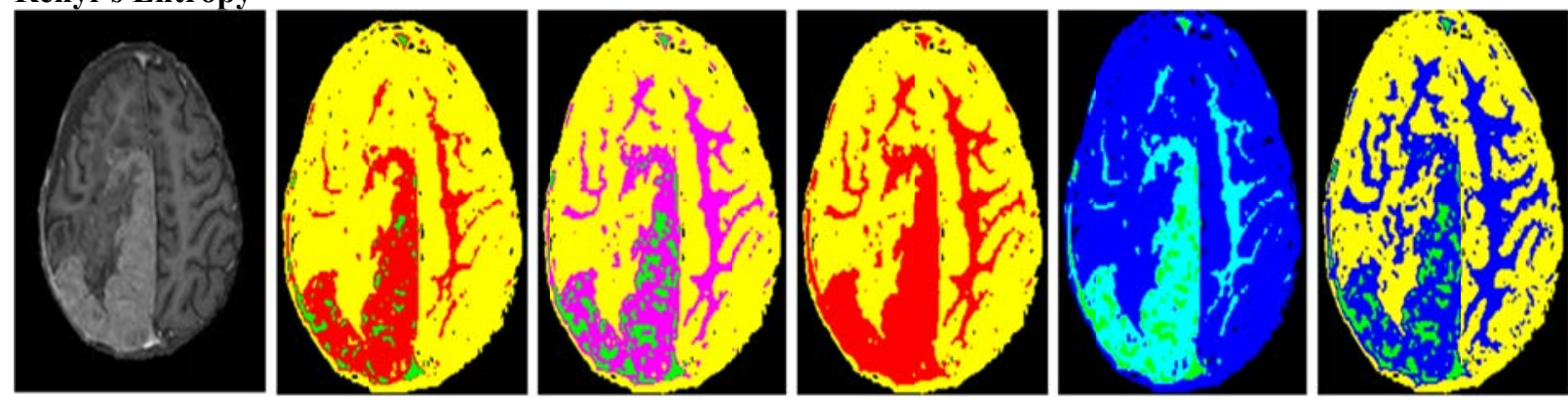

Fig. 9. Primitive Neuro Ectodermal tumour affected brain image and optimal threshold images obtained with five algorithms

\section{PERFORMANCE EVALUATION OF PROPOSED METHOD FOR IMAGE COMPRESSION}

This section explains how the proposed HBFOAPSO is effective in image compression in terms of PSNR, MSE, SSIM and WPSNR.

\section{PEAK SIGNAL TO NOISE RATIO}

Peak signal to noise ratio shows the noise contaminated in reconstructed image. A high value of PSNR shows that effect of noise on reconstructed image is a little low. The highest intensity level of the input gray level MRI brain image is 255 and is indicated with symbol MAX. PSNR value is inversely proportional to MSE and in general the range of PSNR value is between $20 \mathrm{~dB}$ to $40 \mathrm{~dB}$ and is calculated using the Eq. (15).

$$
P S N R=10 \log _{10}\left(\frac{M A X^{2}}{M S E}\right)
$$

From the above equation it is clear that PSNR value increased with the decrement in MSE value.

The bits per pixel (bpp) is the ratio of compressed image size (in terms of bits) $(\tilde{I})$ and total number of pixels in compressed image $\left(\tilde{I}_{T}\right)$. Below table shows the bpp and number of thresholds. All the pixels in the original image are replaced with optimal thresholds, if number of thresholds $\mathrm{Th}=2$, then 2 bits are enough to represent 2 thresholds. So compressed image size (in terms of bits) is $256^{*} 256^{*} 2$ (since original image size is $\left.256^{*} 256\right)$. Therefore bpp $=256 * 256 * \mathrm{Th} / 256^{*} 256 * 8$.

\begin{tabular}{|c|l|}
\hline $\begin{array}{c}\text { Number of } \\
\text { thresholds } \\
(\mathrm{Th})\end{array}$ & $\mathrm{bpp}=\tilde{I} / \tilde{I}_{T}$ \\
\hline 2 & 0.25 \\
\hline 3 & 0.375 \\
\hline 4 & 0.5 \\
\hline 5 & 0.625 \\
\hline
\end{tabular}

Fig. 22 and Fig. 23 shows the bar chart between the PSNR and bits per pixel (bpp), from these figures average peak signal to noise ratio of the proposed HBFOA-PSO is around 32 decibels and this value is far better when compared to other algorithms and its value is higher with Renyi's entropy when compared with the Kapur's entropy. From Fig. 10 to Fig. 15 it is observed that reconstructed image quality of the proposed HBFOA-PSO is better as compared with other algorithms and Fig. 16 to Fig. 21 show the optimal thresholds obtained with different algorithms and all are marked on histogram of respective brain tumour images. 
MEAN SQUARE ERROR: It is the procedure of squaring the predictable quantities. It is the average error between the input and reconstructed image and the result is squared and is calculated using Eq. (16).

$M S E=\frac{1}{M X M} \sum_{i=1}^{M} \sum_{j=1}^{M}\left(I_{i j}-\tilde{I}_{i j}\right)^{2}$

The algorithm which gives lower value of mean squared error is the best algorithm. Lower value of MSE shows less difference between the input image and reconstructed image. In above equation $\mathrm{M}$ is size of the input image, $\mathrm{I}$ is original input image and $\tilde{I}$ is reconstructed image or decompressed image. With the proposed method the value of MSE is lesser when compared to other algorithms.

Fig. 24 and Fig. 25 show the rate distortion curve drawn between different images on x-axis and MSE on $y$-axis by considering bits per pixel (bpp) 0.625 . These figures show the comparison of various MSE obtained with the various algorithms. MSE values are image compression measuring parameter which measures the deformation levels in the reconstructed image and this deformation levels are treated as error and is measured by taking pixel wise difference between input brain image and reconstructed image. As a whole proposed HBFOA-PSO has lower value of MSE when compared with others.

\section{STRUCTURAL SIMILARITY INDEX MEAS-} URE (SSIM)

PSNR and MSE values are measured with respect to intensity level of the input image and reconstructed image. Sometimes these two fail in measuring the reconstructed image visual quality. Sometimes PSNR value obtained with the technique may be high but visual quality is poor, so SSIM is introduced in this paper.The SSIM measures the similarity between input image and reconstructed image with separate luminance (L), contrast (C) and structure (S) components. SSIM of $\mathrm{y}$ and $\bar{y}$ is calculated using following equation

$$
\operatorname{SSIM}(y, \bar{y})=[L(I, \widetilde{I})]^{\alpha}[C(I, \widetilde{I})]^{\beta}[S(I, \widetilde{I})]
$$

$\alpha, \beta$ and $\gamma$ are the adjustable parameters which gives the relative importance of the three components and are equal to one in this paper for effortless calculation of SSIM.

$$
\operatorname{SSIM}=\left(2 \mu_{I} \mu_{\tilde{I}}+C 1\right)\left(2 \sigma_{\tilde{I}}+C 2\right) /\left(\mu_{I}^{2}+\mu_{\tilde{I}}^{2}-C 1\right)\left(\sigma_{I}^{2}+\sigma_{\tilde{I}}^{2}-C 2\right)
$$

Where $\mu_{I}$ and $\mu_{\tilde{I}}$ are the mean value of the original image $I$ and reconstructed image $\tilde{I}, \sigma_{I}$ and $\sigma_{\tilde{I}}$ are the standard deviation of original image $I$ and reconstructed image $\tilde{I}, \sigma_{I I}$ is the cross-correlation and $C 1 \& C 2$ are constants which are equal to 0.065 . The range of SSIM is -1 to +1 and SSIM value equal to one shows original image and reconstructed image is similar. The algorithm is said to be good if SSIM value is near around +1 . Table 2 shows the SSIM of various methods with Renyi's entropy and Kapur's entropy and it shows proposed method SSIM is higher than other methods.

$$
\mu_{I}=\frac{1}{N} \sum_{i=1}^{N} I_{i}
$$

Cross - correlation $=\sigma_{\widetilde{I I}}=\frac{1}{N-1} \sum_{i=1}^{N}\left(I_{i}-\mu_{I}\right)\left(\widetilde{I}_{i}-\mu_{\widetilde{I}}\right)$

\section{WEIGHTED PSNR (WPSNR)}

The major advantage of PSNR is its simplicity in the calculation while major disadvantage is that it does not consider any of the human visual system (HVS) attributes. So there is need of WPSNR which incorporate HVS parameters. WPSNR is HVS-based method and more accurate than PSNR. The WPSNR uses the principle of redundancy of the human eye toward high frequency components in images. The human perception of vision is less sensitive to edges than smooth areas. The WPSNR is nothing but PSNR weighted by the HVS parameter (Navas et al., 2011). The WPSNR in $\mathrm{dB}$ is expressed as

$$
\text { WPSNR }=10 \log _{10}\left(\frac{255^{2}}{\mathrm{NVF} \times \mathrm{MSE}}\right)
$$

Where $N V F$ is noise visibility function and defined as

$$
\mathrm{NVF}=\operatorname{norm}\left(\frac{1}{1+\delta_{\text {block }}^{2}}\right)
$$

Where, $\delta_{\text {block }}$ is the standard deviation of pixels having a specific size $(8 \times 8)$. In smooth regions, the value of NVF is near to zero and in the regions with edges and texture it is near to unity. From Table 2 it is observed that proposed method is better in WPSNR compared to other methods. 


\section{Kapur's Entropy}
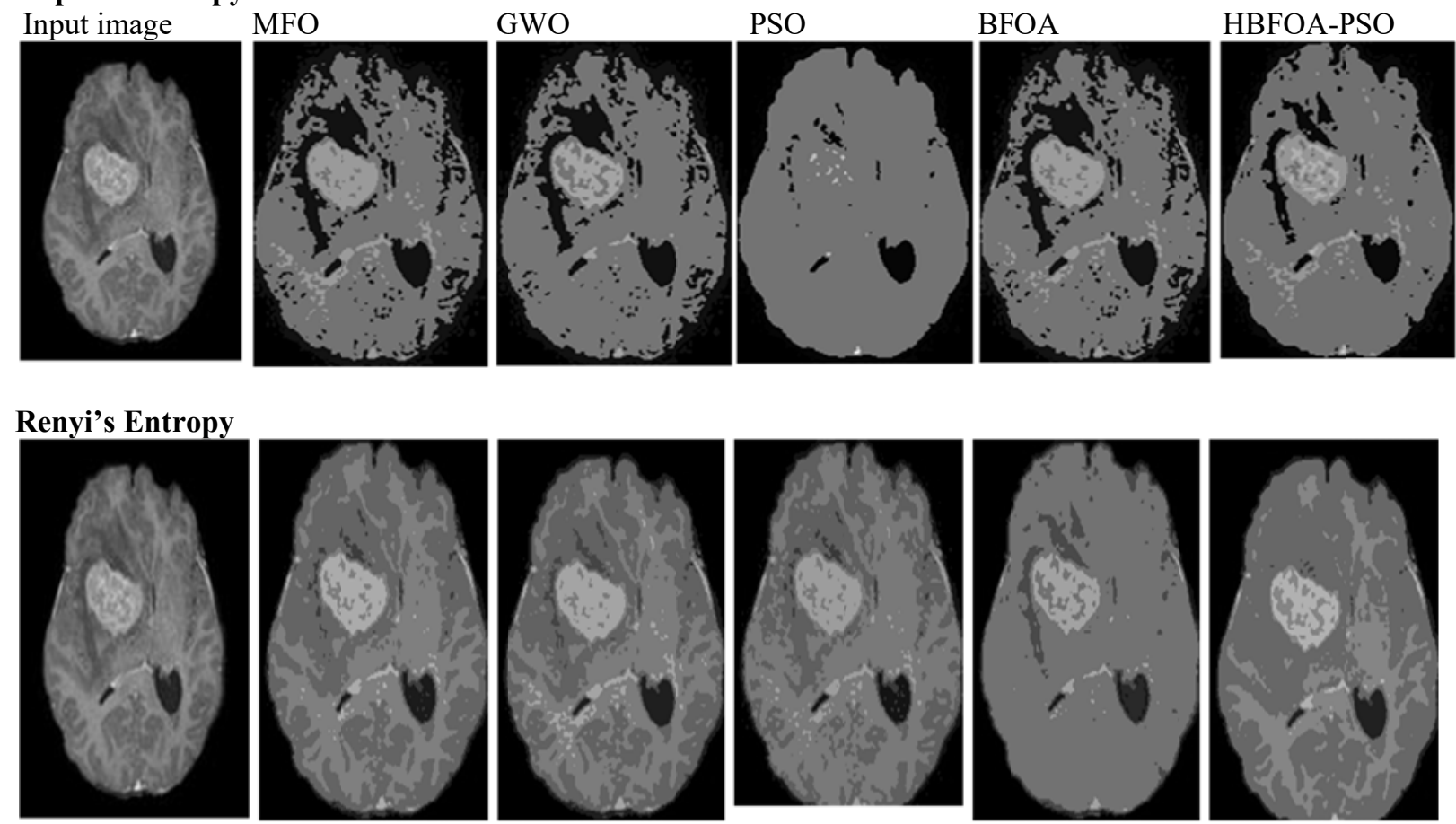

Fig. 10. Astrocytoma tumour affected brain image and decompressed image obtained with five algorithms

\section{Kapur's Entropy}
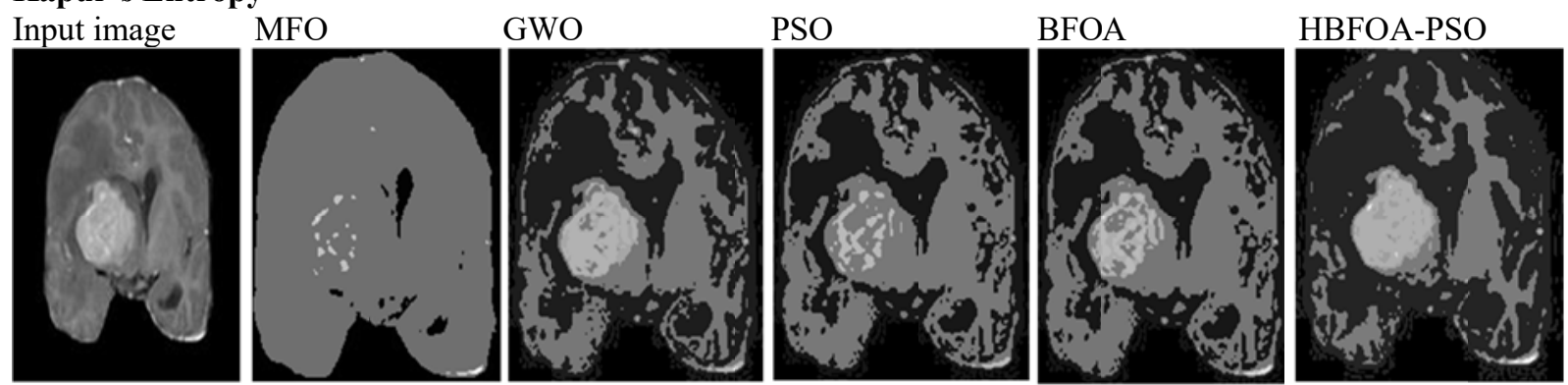

\section{Renyi's Entropy}
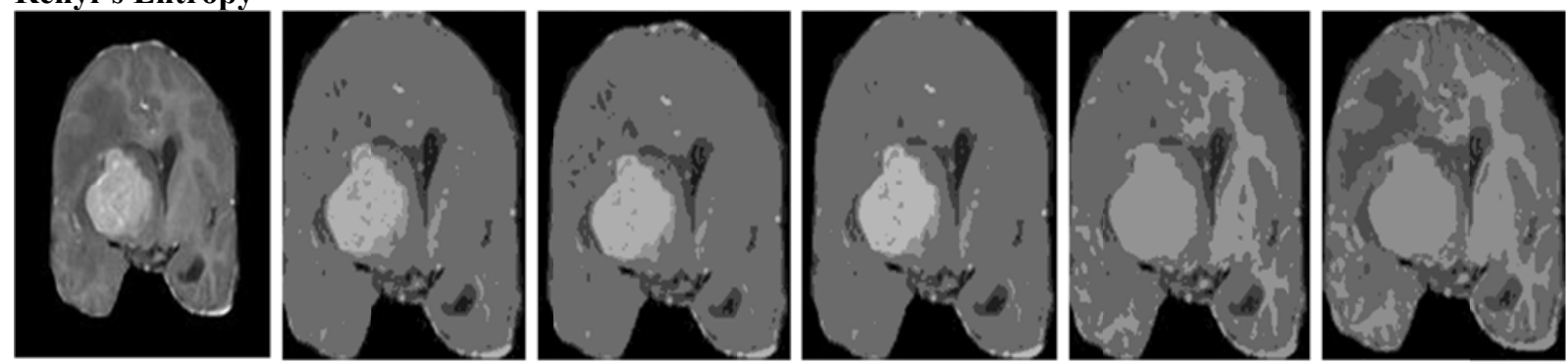

Fig. 11. Coronary T1 Astrocytoma tumour affected brain image and decompressed images obtained with five algorithms 
Kapur's Entropy
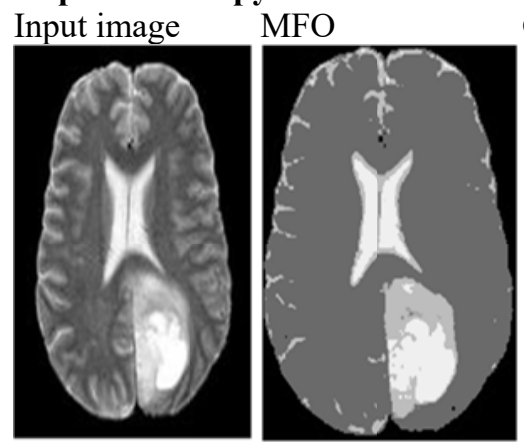

GWO
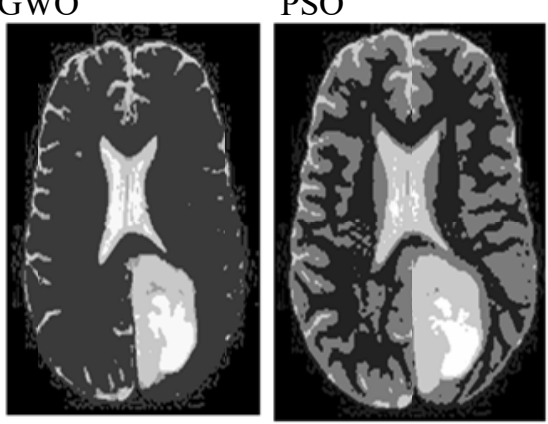

BFOA
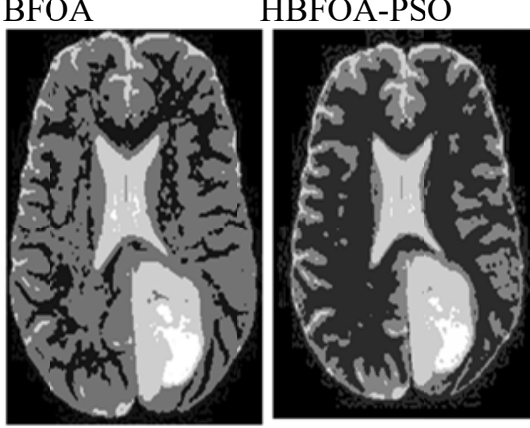

\section{Renyi's Entropy}
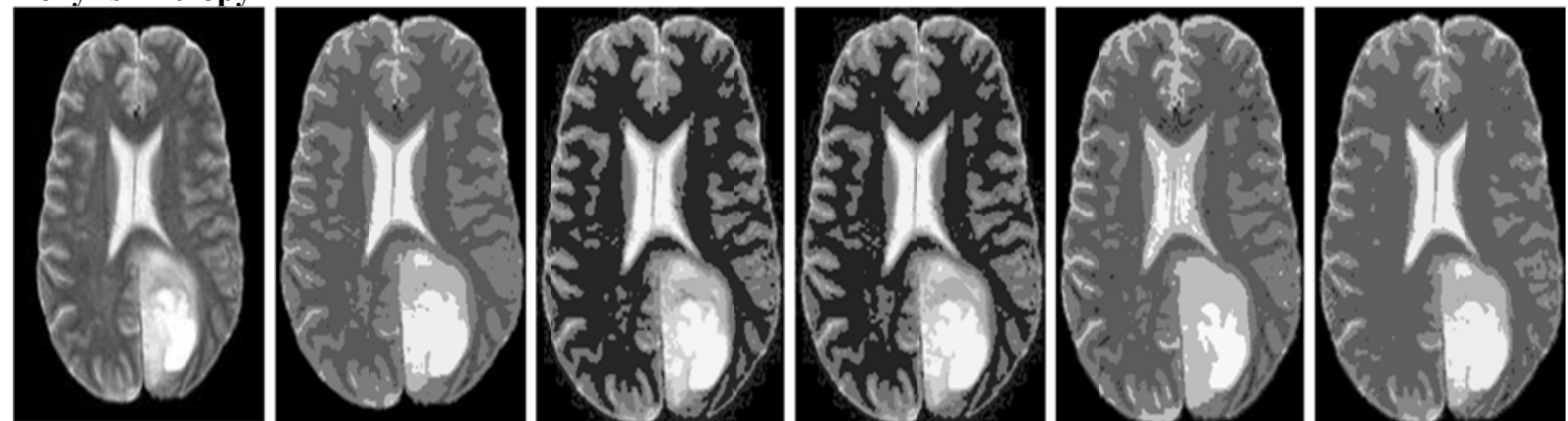

Fig. 12. Glioma affected brain image and decompressed images obtained with five algorithms

\section{Kapur's Entropy}
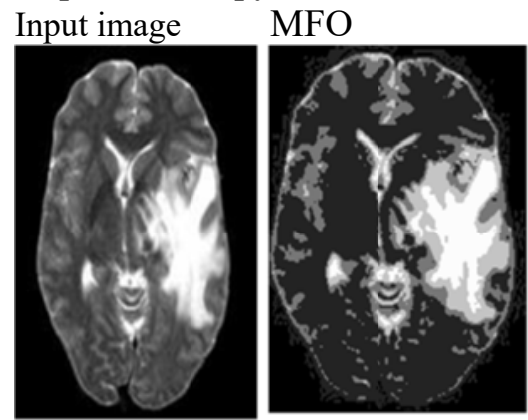

GWO
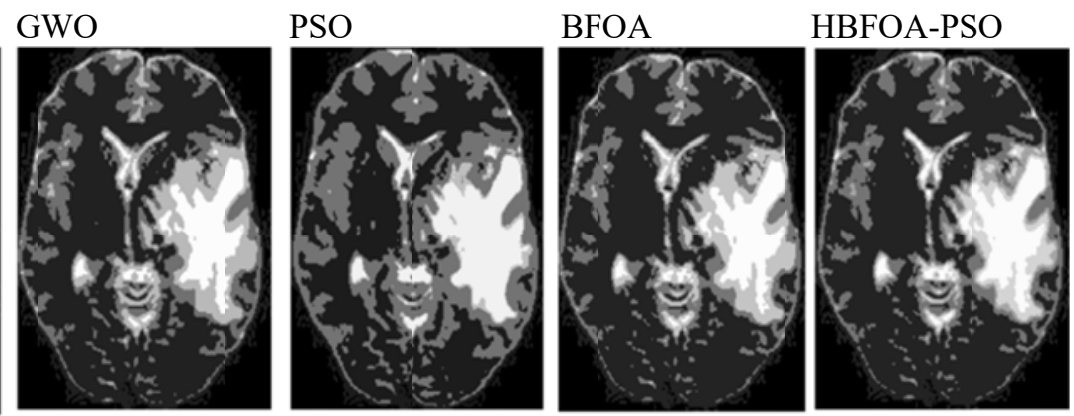

\section{Renyi's Entropy}
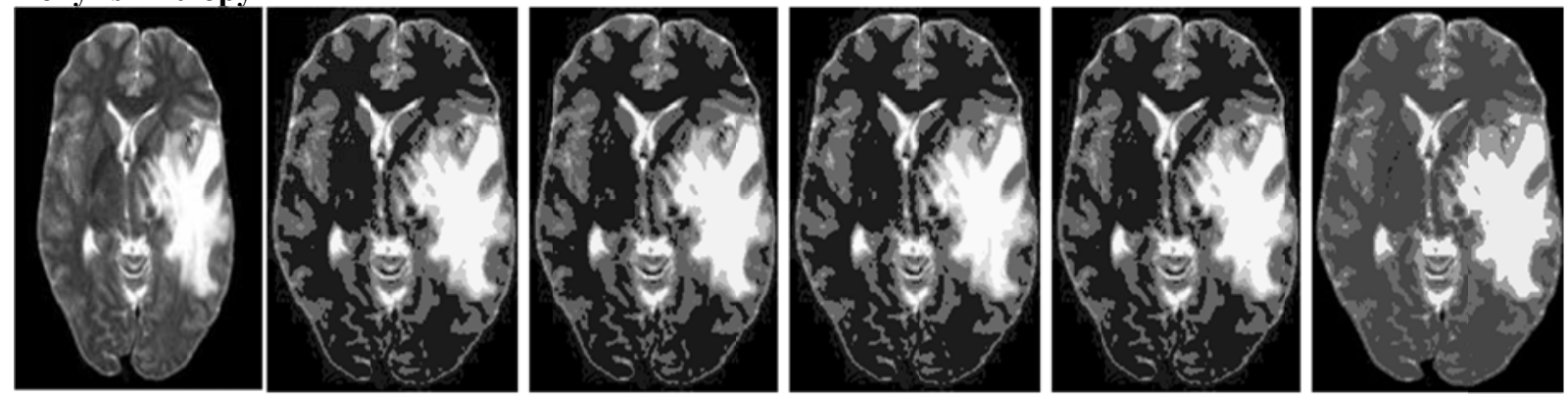

Fig. 13. Metastatic Bronchogenic Carcinoma affected brain image and decompressed images obtained with five algorithms 


\section{Kapur's Entropy}

Input image

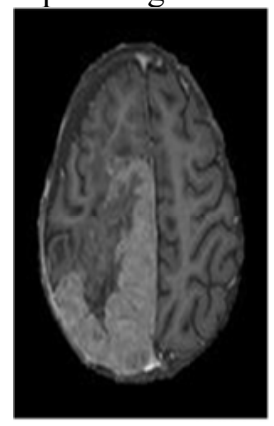

MFO

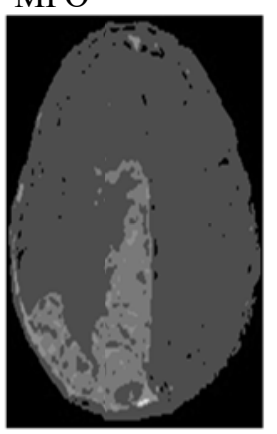

GWO

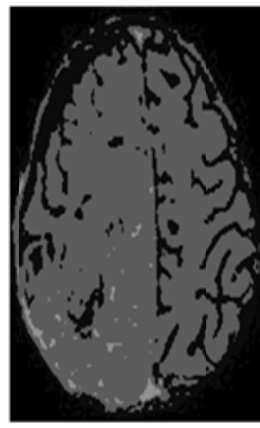

PSO

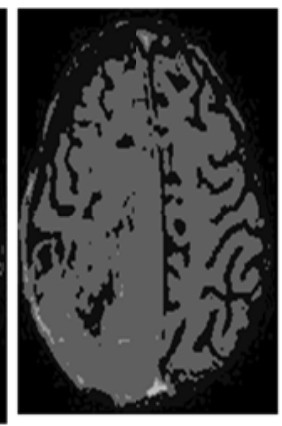

BFOA

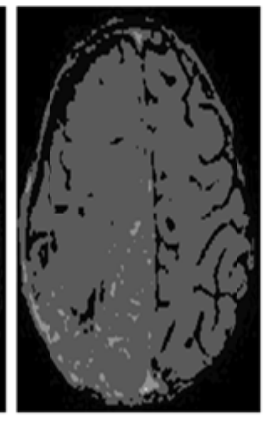

HBFOA-PSO

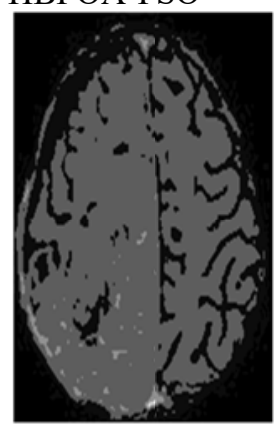

\section{Renyi's Entropy}
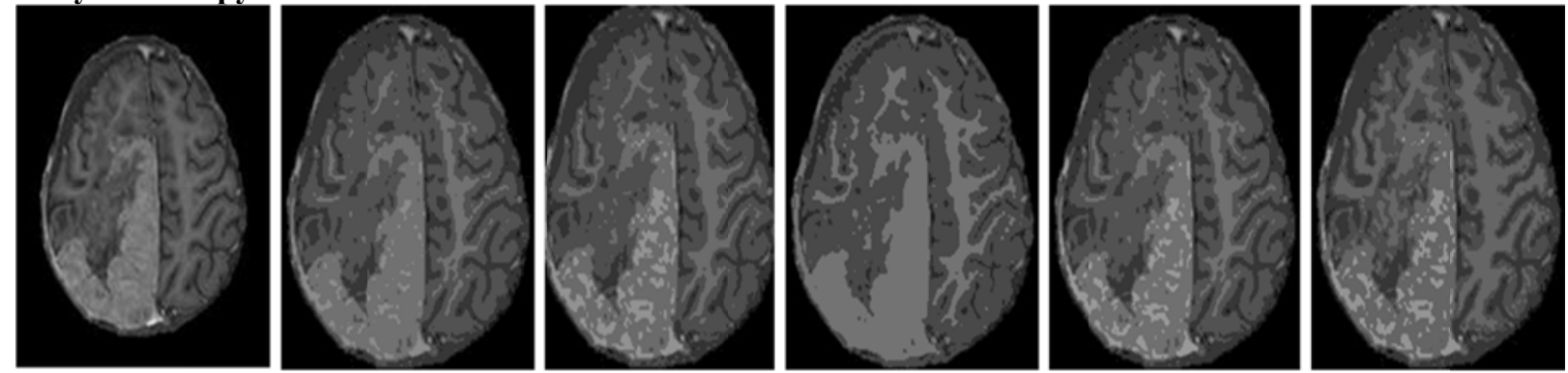

Fig. 14. Primitive Neuro Ectodermal tumour affected brain image and decompressed images obtained with five algorithms

\section{Kapur's Entropy}
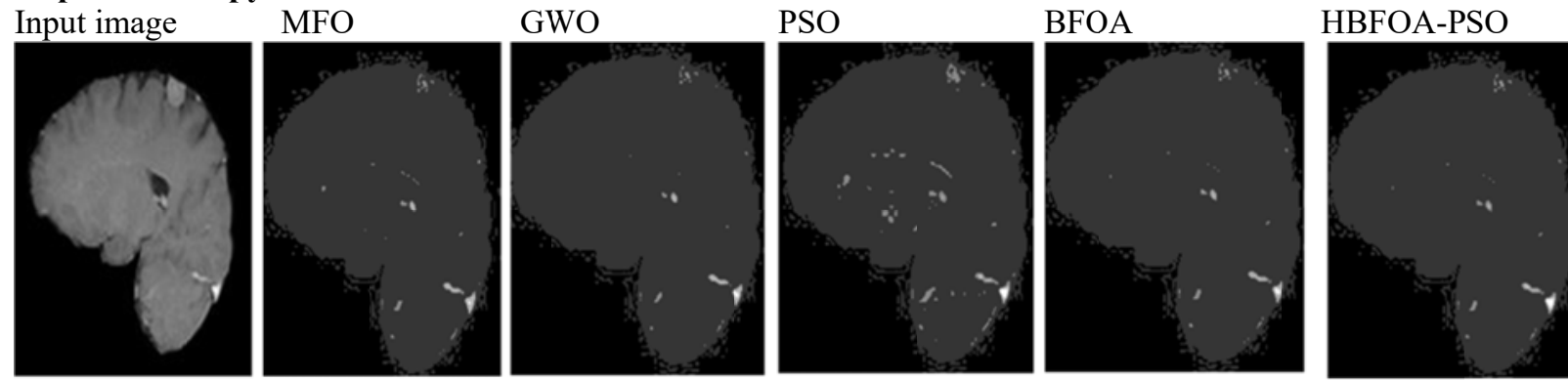

\section{Renyi's Entropy}
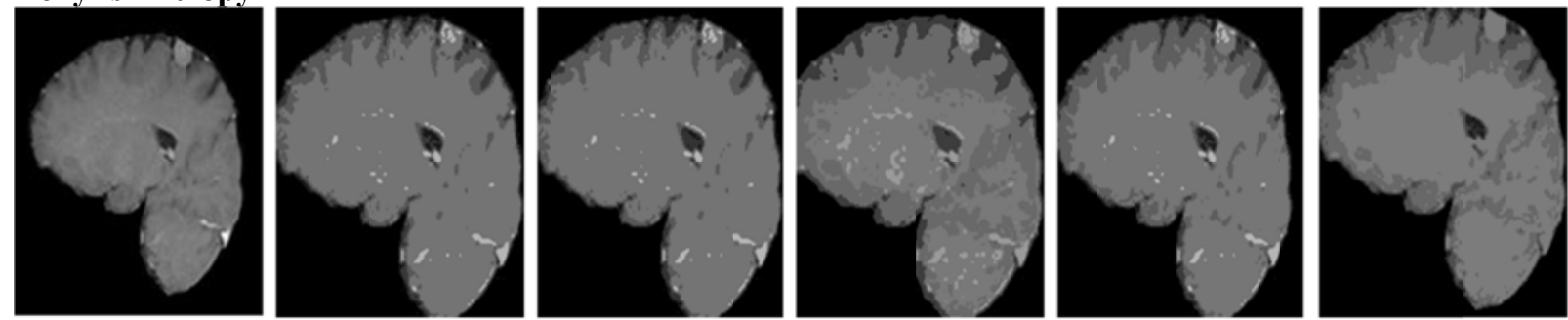

Fig. 15. Meningioma affected brain image and decompressed images obtained with five algorithms 

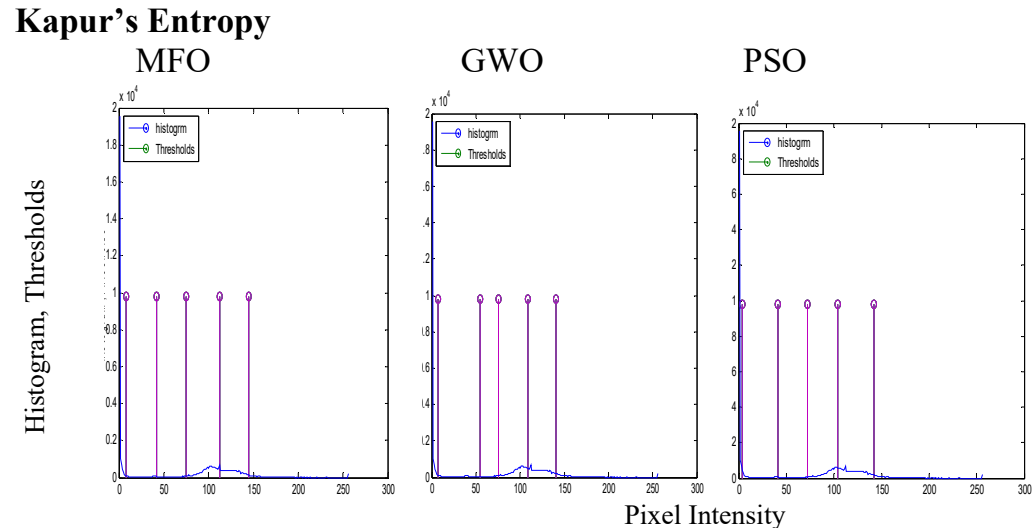

BFOA

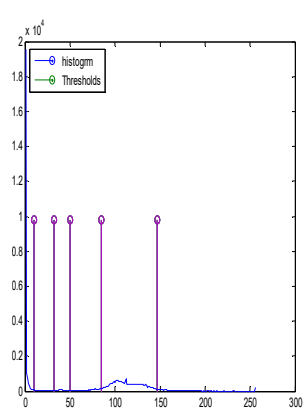

HBFOA-PSO

\section{Renyi's Entropy}
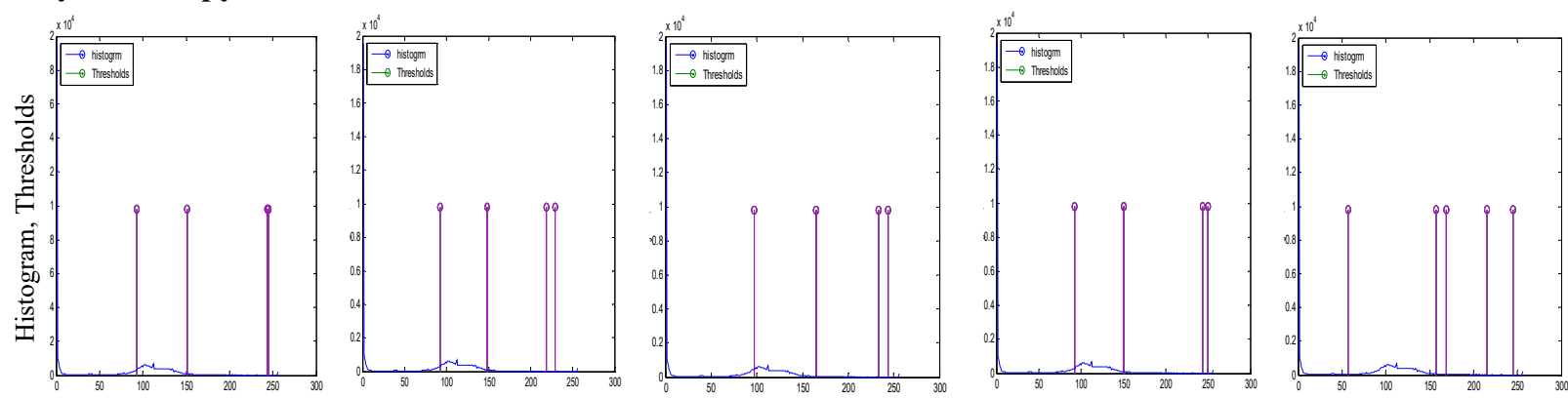

Pixel Intensity

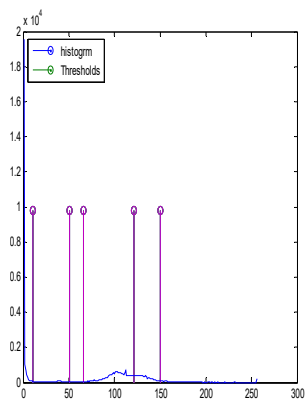

Fig. 16. Optimal thresholds on Histogram of Astrocytoma tumour affected brain image with five algorithms

\section{Kapur's Entropy}

MFO

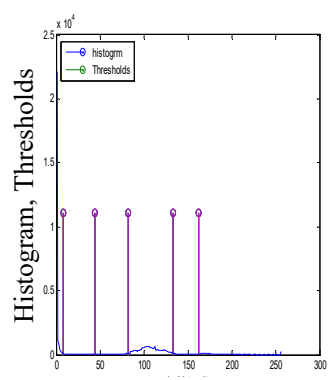

Renyi's Entropy
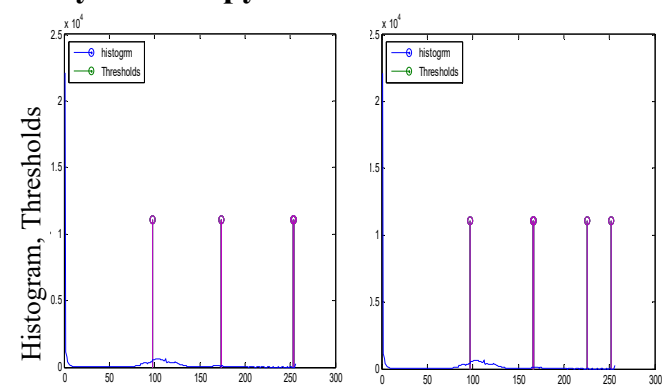

PSO

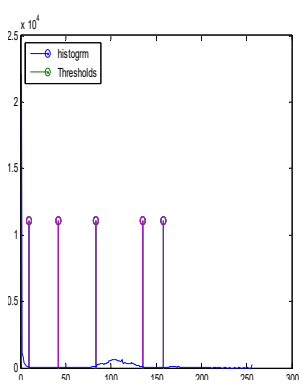

Pixel Intensity
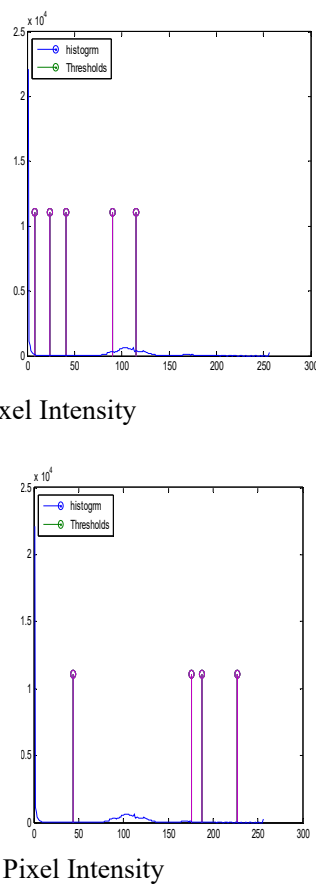

BFOA
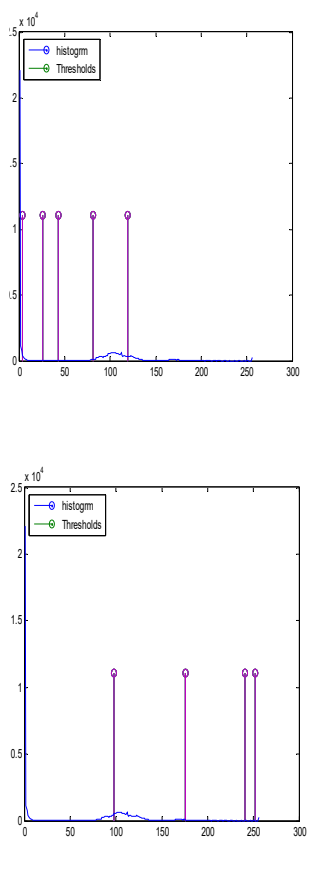

HBFOA-PSO
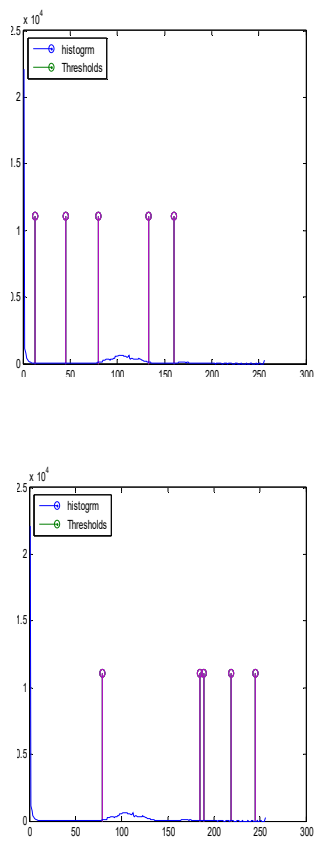

Fig. 17. Optimal thresholds on Histogram of Coronary T1 Astrocytoma tumour affected brain image with five algorithms 


\section{Kapur's Entropy} MFO

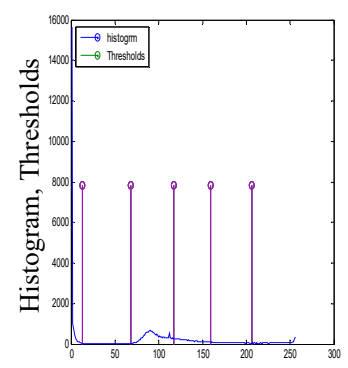

\section{Renyi's Entropy}
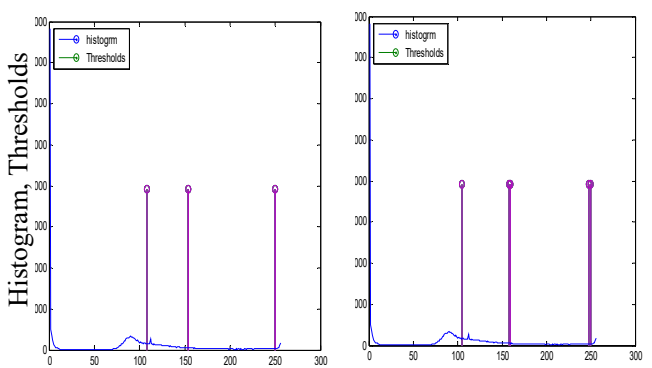

Pixel Intensity

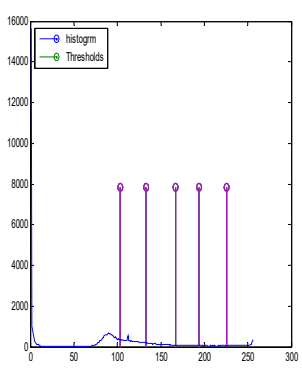

PSO

Pixel Intensity
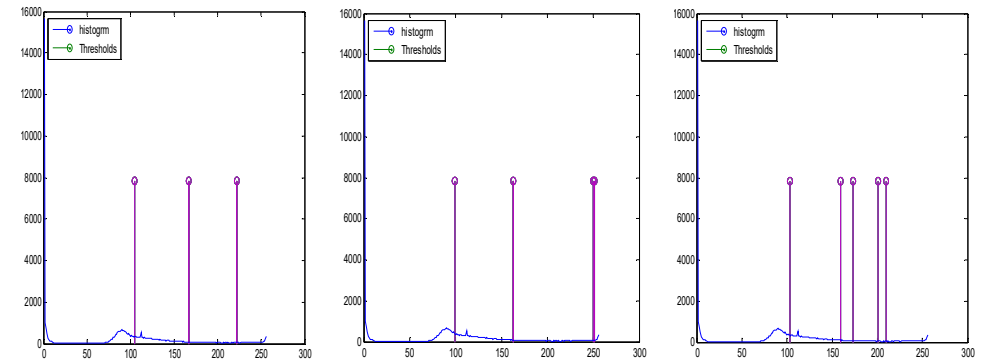

HBFOA-PSO

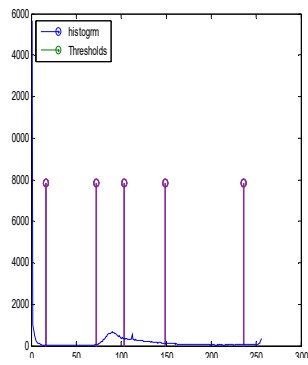

Fig. 18. Optimal thresholds on Histogram of Glioma affected brain image with five algorithms

\section{Kapur's Entropy}
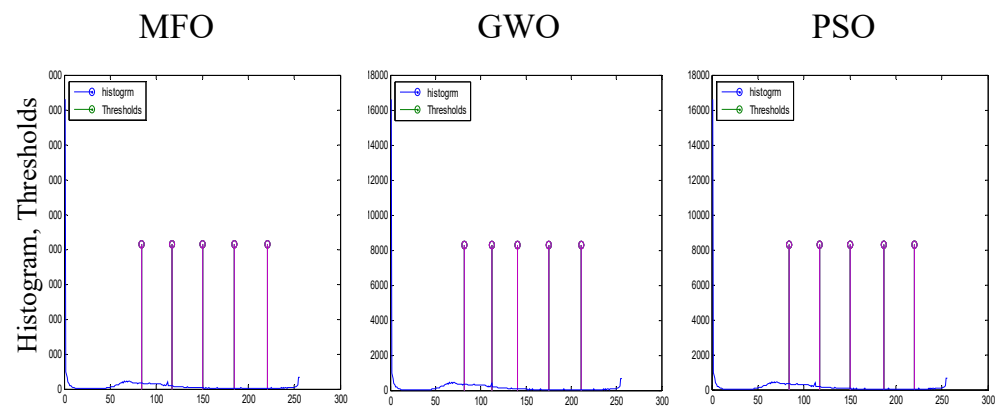

BFOA

HBFOA-PSO

\section{Renyi's Entropy}
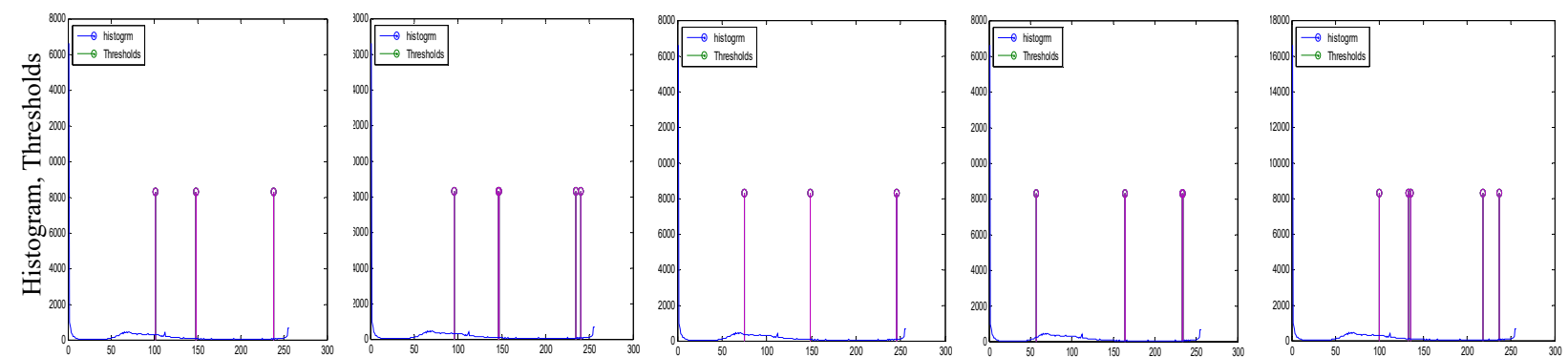

Pixel Intensity

Fig. 19. Optimal thresholds on Histogram of Metastatic Bronchogenic Carcinoma affected brain image with five algorithms 


\section{Kapur's Entropy}

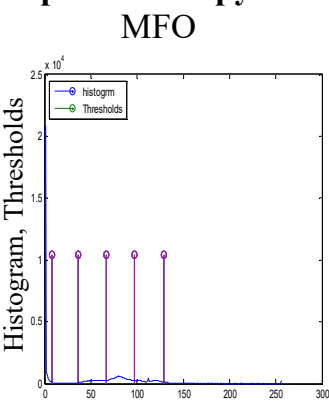

Renyi's Entropy
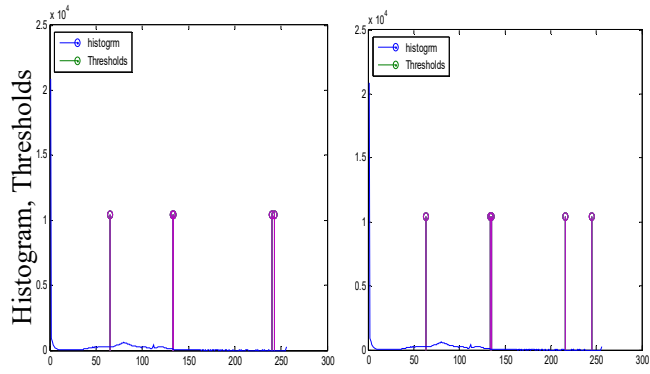

Pixel Intensity

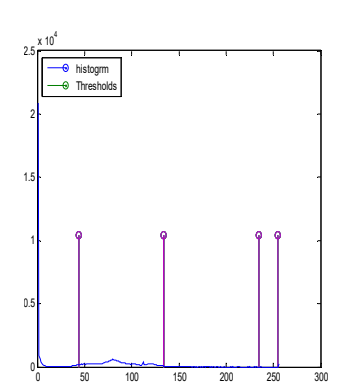

PSO

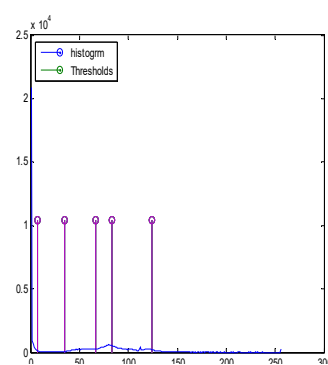

Pixel Intensity
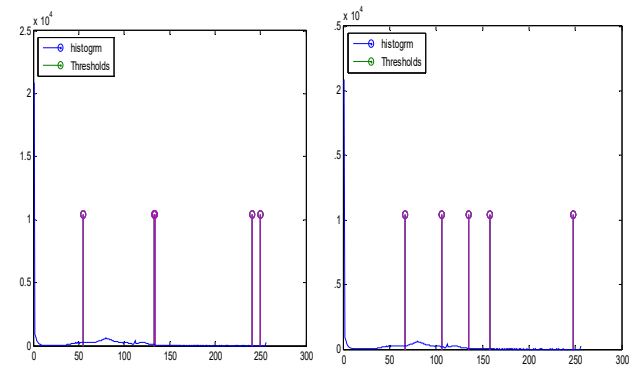

Fig. 20. Optimal thresholds on Histogram of Primitive Neuro Ectodermal tumour affected brain image with five algorithms

\section{Kapur's Entropy}
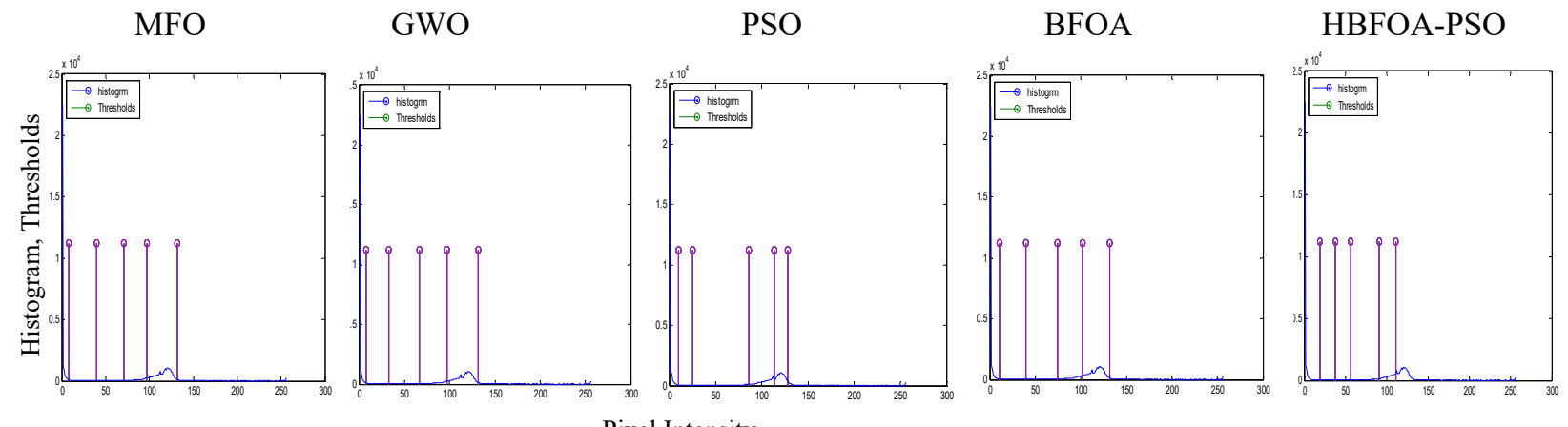

\section{Renyi's Entropy}
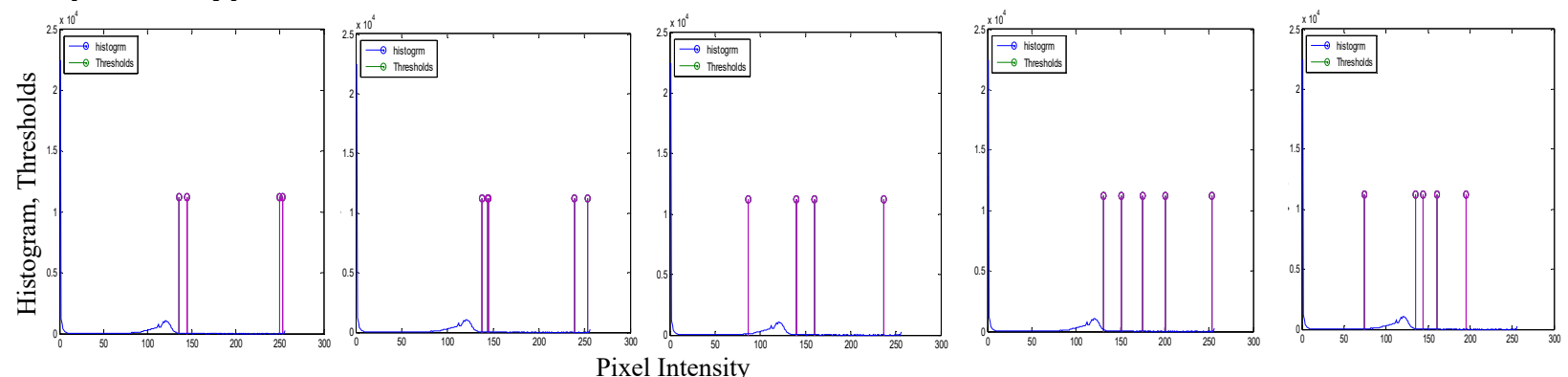

Fig. 21. Optimal thresholds on Histogram of Meningioma affected brain image with five algorithms 
Table 1. Evaluation of Fitness, Mean, Standard deviation and Elapsed time of five methods for brain images

\begin{tabular}{|c|c|c|c|c|c|c|c|c|c|}
\hline \multirow[t]{2}{*}{ Image } & \multirow{2}{*}{$\begin{array}{c}\text { Optimization } \\
\text { Technique } \\
\end{array}$} & \multicolumn{2}{|c|}{ Fitness value } & \multicolumn{2}{|c|}{ Mean } & \multicolumn{2}{|c|}{ Standard deviation } & \multicolumn{2}{|c|}{$\begin{array}{l}\text { Elapsed time } \\
\quad(\mathrm{sec})\end{array}$} \\
\hline & & Kapur & Renyi & Kapur & Renyi & Kapur & Renyi & Kapur & Renyi \\
\hline \multirow{5}{*}{ Meningioma } & GWO & 9.8524 & 16.172 & 10.465 & 16.904 & 0.08711 & 0.117654 & 28.931 & 10.809 \\
\hline & MFO & 10.391 & 16.631 & 10.483 & 16.922 & 0.0001 & $3.61 \mathrm{E}-15$ & 9.0867 & 7.2405 \\
\hline & $\mathrm{PSO}$ & 10.483 & 16.913 & 10.055 & 15.99 & 0.19127 & 0.410305 & 19.165 & 9.2245 \\
\hline & BFOA & 10.485 & 16.922 & 10.485 & 16.913 & $5.42 \mathrm{E}-15$ & $1.45 \mathrm{E}-14$ & 8.0601 & 7.0205 \\
\hline & HBFOA-PSO & 10.537 & 16.968 & 9.8524 & 16.172 & $3.61 \mathrm{E}-15$ & $3.61 \mathrm{E}-15$ & 39.543 & 57.999 \\
\hline \multirow{5}{*}{ PNET } & GWO & 10.81 & 15.971 & 10.81 & 15.971 & 0.03428 & 0.144739 & 20.027 & 16.979 \\
\hline & MFO & 11.149 & 16.912 & 11.088 & 16.122 & $5.42 \mathrm{E}-15$ & $3.61 \mathrm{E}-15$ & 10.23 & 7.1725 \\
\hline & PSO & 11.176 & 17.015 & 11.149 & 17.015 & 0.0982 & 0.569382 & 55.656 & 9.8013 \\
\hline & BFOA & 11.183 & 17.075 & 11.172 & 17.02 & $5.42 \mathrm{E}-15$ & $1.08 \mathrm{E}-14$ & 13.103 & 7.6018 \\
\hline & HBFOA-PSO & 11.187 & 17.086 & 11.176 & 17.075 & $5.42 \mathrm{E}-15$ & $7.23 \mathrm{E}-15$ & 40.608 & 59.407 \\
\hline \multirow{5}{*}{ Metastatic } & GWO & 12.191 & 19.011 & 12.191 & 19.011 & 0.03701 & 0.038206 & 18.119 & 15.381 \\
\hline & MFO & 12.518 & 19.704 & 12.518 & 19.419 & $9.03 \mathrm{E}-15$ & $1.08 \mathrm{E}-14$ & 21.278 & 10.68 \\
\hline & PSO & 12.546 & 19.711 & 12.34 & 19.711 & 0.12774 & 0.22731 & 19.185 & 94.153 \\
\hline & BFOA & 12.55 & 19.762 & 12.535 & 19.762 & $9.03 \mathrm{E}-15$ & 0.0909 & 9.7452 & 9.4357 \\
\hline & HBFOA-PSO & 12.551 & 19.765 & 12.551 & 19.749 & $1.81 \mathrm{E}-15$ & 0.09090 & 38.520 & 48.261 \\
\hline \multirow{5}{*}{ Glioma } & GWO & 12.002 & 19.041 & 12.002 & 19.041 & 0.03799 & 0.05544 & 17.759 & 12.135 \\
\hline & MFO & 12.36 & 19.634 & 12.159 & 19.634 & $5.42 \mathrm{E}-15$ & $1.08 \mathrm{E}-14$ & 12.25 & 7.8565 \\
\hline & PSO & 12.363 & 19.658 & 12.368 & 19.658 & 0.12408 & 0.20444 & 38.2 & 9.9885 \\
\hline & BFOA & 12.394 & 19.824 & 12.36 & 19.501 & $9.03 \mathrm{E}-15$ & $3.61 \mathrm{E}-15$ & 9.8131 & 17.692 \\
\hline & HBFOA-PSO & 12.395 & 19.835 & 12.363 & 19.805 & 0.0909 & $3.61 \mathrm{E}-15$ & 45.879 & 52.848 \\
\hline \multirow{5}{*}{$\begin{array}{l}\text { Coronary T1 } \\
\text { Astrocytoma }\end{array}$} & GWO & 10.881 & 16.965 & 10.881 & 16.965 & 0.03465 & 0.063441 & 25.367 & 16.936 \\
\hline & MFO & 11.027 & 18.248 & 10.953 & 18.248 & 0.0075 & $3.61 \mathrm{E}-15$ & 8.4109 & 9.0397 \\
\hline & PSO & 11.102 & 18.268 & 11.027 & 18.268 & 0.12238 & 0.341585 & 17.462 & 10.561 \\
\hline & $\mathrm{BFOA}$ & 11.156 & 18.273 & 11.102 & 17.681 & $3.61 \mathrm{E}-15$ & 7.23E-15 & 12.135 & 8.3687 \\
\hline & HBFOA-PSO & 11.165 & 18.348 & 11.136 & 18.3 & $1.81 \mathrm{E}-15$ & $3.61 \mathrm{E}-15$ & 36.64 & 58.186 \\
\hline \multirow{5}{*}{ Astrocytoma } & GWO & 10.736 & 17.244 & 11.011 & 18.052 & 0.02483 & 0.078797 & 17.74 & 21.74 \\
\hline & MFO & 11.022 & 17.635 & 11.024 & 17.825 & $1.81 \mathrm{E}-15$ & 3.61E-15 & 7.577 & 8.0546 \\
\hline & $\mathrm{PSO}$ & 11.024 & 17.825 & 10.861 & 17.543 & 0.13426 & 0.424639 & 16.351 & 13.634 \\
\hline & BFOA & 11.024 & 18.04 & 11.024 & 17.635 & $3.61 \mathrm{E}-15$ & $3.61 \mathrm{E}-15$ & 14.347 & 7.9109 \\
\hline & HBFOA-PSO & 11.025 & 18.091 & 10.736 & 17.244 & $1.81 \mathrm{E}-15$ & $7.23 \mathrm{E}-15$ & 50.755 & 65.194 \\
\hline
\end{tabular}


Table 2. Evaluation of SSIM and WPSNR of five methods for brain images

\begin{tabular}{|c|c|c|c|c|c|}
\hline \multirow[t]{2}{*}{ Image } & \multirow{2}{*}{$\begin{array}{c}\text { Optimization } \\
\text { Technique }\end{array}$} & \multicolumn{2}{|c|}{ SSIM } & \multicolumn{2}{|c|}{ WPSNR (dB) } \\
\hline & & Kapur & Renyi & Kapur & Renyi \\
\hline \multirow{5}{*}{ Meningioma } & GWO & 0.658670 & 0.886195 & 32.04823 & 15.609031 \\
\hline & MFO & 0.667427 & 0.904109 & 32.058787 & 15.937439 \\
\hline & PSO & 0.667626 & 0.904584 & 32.063169 & 16.052494 \\
\hline & BFOA & 0.667959 & 0.904651 & 32.069061 & 18.125513 \\
\hline & HBFOA-PSO & 0.667986 & 0.910922 & 32.108383 & 19.708357 \\
\hline \multirow{5}{*}{ PNET } & GWO & 0.6827744 & 0.832106 & 19.053239 & 18.153405 \\
\hline & MFO & 0.7056383 & 0.8752121 & 23.705779 & 18.531831 \\
\hline & PSO & 0.7226091 & 0.8762945 & 24.686436 & 18.943262 \\
\hline & BFOA & 0.7412038 & 0.8776707 & 27.591976 & 19.081611 \\
\hline & HBFOA-PSO & 0.7586052 & 0.8814224 & 27.983886 & 20.746489 \\
\hline \multirow{5}{*}{ Metastatic } & GWO & 0.6717987 & 0.7295003 & 27.416015 & 17.666669 \\
\hline & MFO & 0.6747454 & 0.7368438 & 27.788892 & 26.471868 \\
\hline & PSO & 0.6803707 & 0.7369216 & 27.863482 & 26.496026 \\
\hline & BFOA & 0.6814665 & 0.740371 & 28.048519 & 26.500085 \\
\hline & HBFOA-PSO & 0.6953937 & 0.8511379 & 28.21856 & 26.725894 \\
\hline \multirow{5}{*}{ Glioma } & GWO & 0.5983156 & 0.6936872 & 22.066536 & 16.974562 \\
\hline & MFO & 0.6161177 & 0.6949095 & 27.936855 & 17.619167 \\
\hline & $\mathrm{PSO}$ & 0.6312582 & 0.8396407 & 28.838197 & 18.795318 \\
\hline & BFOA & 0.6353784 & 0.841043 & 29.477103 & 28.513004 \\
\hline & HBFOA-PSO & 0.7363141 & 0.8439056 & 30.557768 & 28.723952 \\
\hline \multirow{5}{*}{$\begin{array}{l}\text { Coronary T1 } \\
\text { Astrocytoma }\end{array}$} & GWO & 0.6350157 & 0.8571572 & 22.77016 & 17.628194 \\
\hline & MFO & 0.6386655 & 0.8581259 & 29.271111 & 17.852049 \\
\hline & $\mathrm{PSO}$ & 0.6450002 & 0.8588663 & 29.412006 & 18.156052 \\
\hline & BFOA & 0.6486089 & 0.8625398 & 29.809665 & 19.952415 \\
\hline & HBFOA-PSO & 0.8092489 & 0.8648337 & 30.562705 & 20.525345 \\
\hline \multirow{5}{*}{ Astrocytoma } & GWO & 0.6304807 & 0.7852364 & 22.866723 & 17.079014 \\
\hline & MFO & 0.6307575 & 0.7952396 & 24.701479 & 17.632169 \\
\hline & $\mathrm{PSO}$ & 0.6566649 & 0.8541937 & 27.212897 & 18.608922 \\
\hline & BFOA & 0.7165008 & 0.8649524 & 29.294284 & 19.586243 \\
\hline & HBFOA-PSO & 0.7276255 & 0.8665126 & 29.294394 & 19.822887 \\
\hline
\end{tabular}



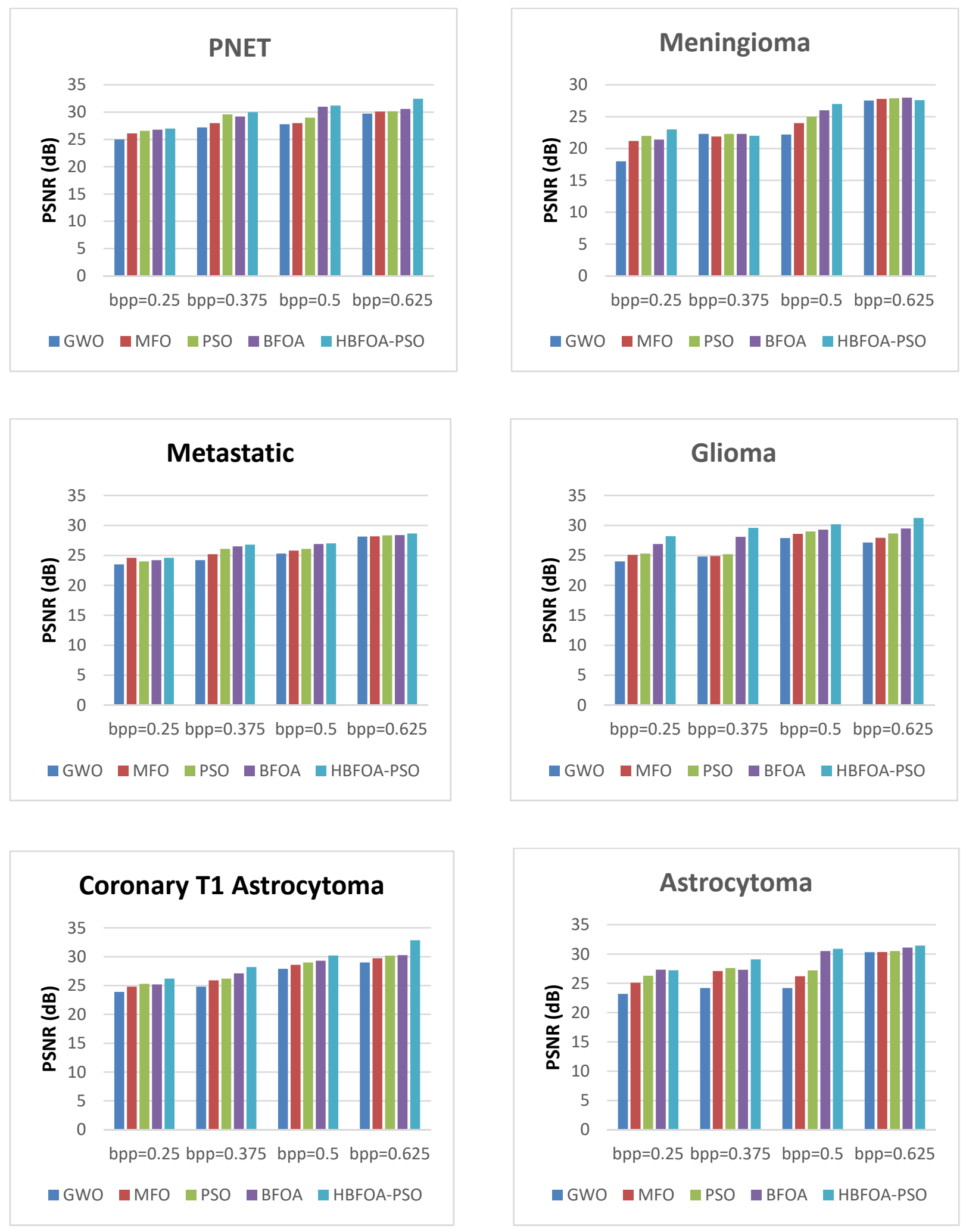

Fig. 22. Peak Signal-to-Noise Ratio of brain images obtained with five algorithms using Renyi Entropy 

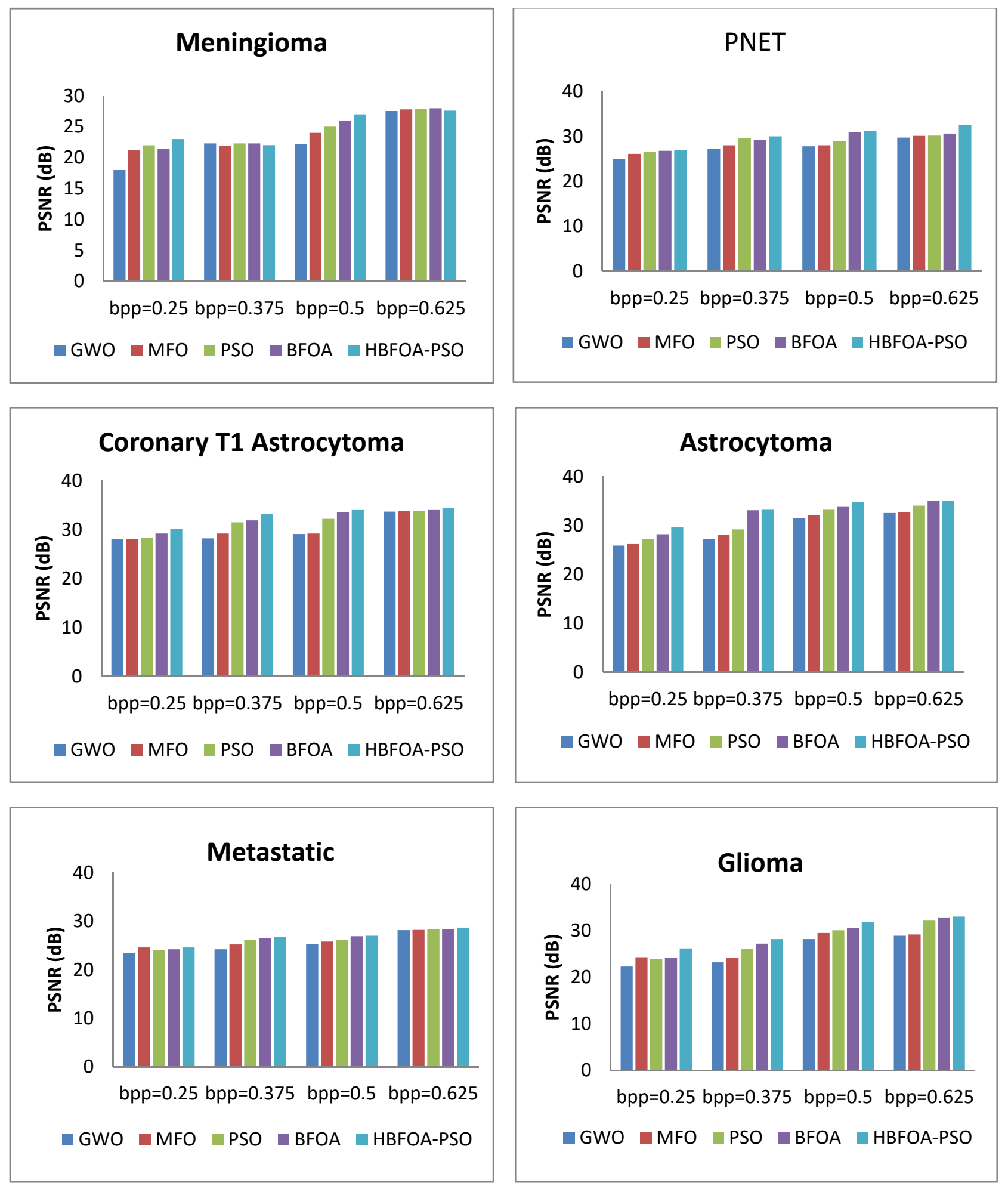

Fig. 23. Peak Signal-to-Noise Ratio of MRI brain images obtained with five algorithms using Kapur's Entropy 


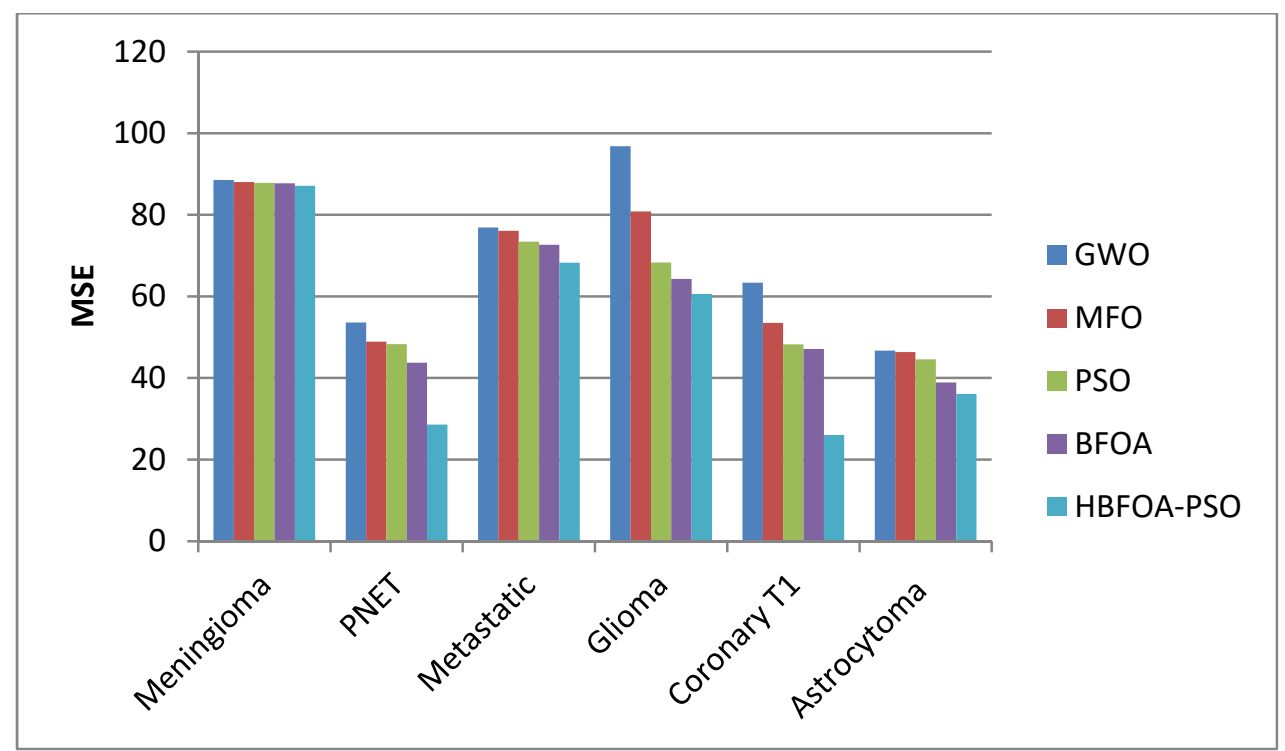

Fig. 24. Rate distortion curve for different images with Kapur's Entropy at bits per pixel (bpp) 0.625

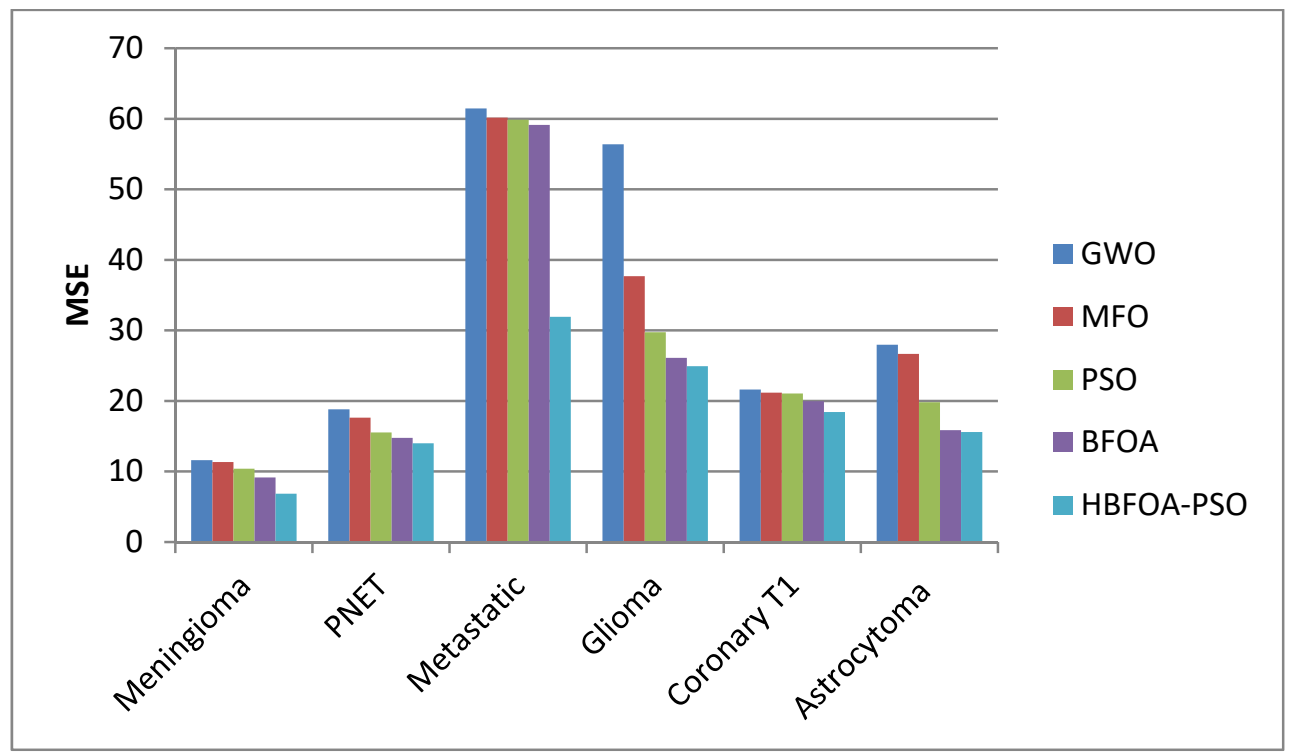

Fig. 25. Rate distortion curve for different images with Renyi Entropy at bits per pixel (bpp) 0.625

\section{CONCLUSIONS}

Numerous experiments and design benchmarks, based on the comparison of HBFOA-PSO with other algorithms, established that it is highly efficient with an almost exponential convergence rate for solving optimization problems. Therefore, HBFOA-PSO algorithm is a trustworthy technique for solving complicated problems in the process of image compression. This study enumerates how the HBFOAPSO uses the Renyi's entropy and Kapur's entopy for optimal thresholds, hence better identification of tumour regions and better reconstructed visual image quality. The HBFOA-PSO algorithm is implemented for compression of MRI brain images. All the results of five experiments established that the HBFOA-PSO algorithm will increase the fidelity of reconstructed images compared to other four methods. Moreover the results of experiments established that the proposed HBFOA-PSO algorithm is capable of identifying the brain tumour regions and have better PSNR, MSE, SSIM and WPSNR when compared with other algorithms. Finally, the performance of the proposed HBFOA-PSO algorithm is found to be better with Renyi's entropy when compared with the Kapur's entropy. 


\section{REFERENCES}

Anitha V, Pallikonda R, Vishnuvarthanan G, Yudong Z, Arunprasath T (2017). An automated hybrid approach using clustering and nature inspired optimization technique for improved tumour and tissue segmentation in magnetic resonance brain images, Appl Soft Comput, 57: 399-26.

Badri NS, Veerakumar T, Esakkirajan SA (2016). Tumour or abnormality identification from magnetic resonance images using statistical region fusion based segmentation, Magn Reson Imaging, 34(9): 1292-304.

Chen-Kuei Y, Wen-Hsiang. T (2015). Color image compression using quantization, thresholding, and edge detection techniques all based on the momentpreserving principle, Pattern Recog Lett, 19: 205-15.

Diego O, Salvador H, Erik C, Gonzalo P, Omar A, Jorge G (2017). Cross entropy based thresholding for magnetic resonance brain images using Crow Search Algorithm, Expert Syst Appl, 79: 164-80

GS Rao, PMK Prasad, DYV Prasad (2016). Performance Analysis of orthogonal and Biorthogonal wavelets for Edge detection of X-ray Images, Procedia Comput Sci, $87: 116-21$.

Irem EK, Ayca Çi, Emine GS, Turgay I (2017). PCA based clustering for brain tumour segmentation of T1W MRI images, Comput Meth Prog Bio, 140: 1928.

Ivan C, Iker G (2017). MRI segmentation fusion for brain tumour detection, Inform Fusion, 36: 1-9.

Kaur LS Gupta RC, Chauhan SCS (2007) Medical ultrasound image compression using joint optimization of thresholding quantization and best-basis selection of wavelet packets, Digit Signal Process, 17: 189-98.

Kaveh Ahmadi, Ahmad YJ, Ezzatollah S (2015). An efficient compression scheme based on adaptive thresholding in wavelet domain using particle swarm optimization, Signal Process-Image, 32: 33-39.

Kennedy J, Eberhart RC (1995). A new Optimizer using Particle Swarm Theory, in Proceedings of Sixth International Symposium on Micro Machine and Human Science, Nagoya, Japan, 6: 39-43.

Lubna F, Adeel Y and Rana HR (2017). Adaptive stochastic segmentation via energy-convergence for brain tumour in MR image., J Vis Commun Image R, 46: 303-11.

Navas KA, Gayathri Devi KG, Athulya MS, Anjali V (2011). MWPSNR: A new image fidelity metric, IEEE Recent Advances in Intelligent Computational Systems (RAICS), 627-32.

Nooshin N, Miroslav K (2017). Automatic tumour segmentation in single-spectral MRI using a texturebased and contour-based algorithm, Expert Syst Appl, 77: $1-10$.
Passino KM (2002). Biomimicry of bacterial foraging for distributed optimization and control, IEEE Control Systems Magazine, 22: 52-67.

Prasanna S, Carrye W, Jerry Y (1997). Threshold Selection Using Renyi's Entropy, Pattern Recogn, 30(1): 71-84.

Prashant S, Ioana M (2003). Selective Thresholding in Wavelet Image Compression, Wavelets and Signal Processing Part of the series Applied and Numerical Harmonic Analysis, 2: 377-81.

Rajeswari R (2012). Type-2 Fuzzy Thresholded Bandlet Transform for Image Compression, Procedia Engineer, 38: 385-90.

Rajinikanth V, Suresh CS, Steven LF, Nachiappan S (2017). Entropy based segmentation of tumour from brain MR images - a study with teaching learning based optimization, Pattern Recog Lett, 94: 87-95.

Ramakrishnan T, Sankaragomathi B (2017). A professional estimate on the computed tomography brain tumour images using SVM-SMO for classification and MRG-GWO for segmentation, ttern Recog Lett, 15:163-71.

Salleh. MFM, Soraghan J (2007). A New Multistage Lattice Vector Quantization with Adaptive Subband Thresholding for Image Compression, EURASIP Journal on Advances in Signal Processing December, 2: $23-34$.

Sathya PD, Kayalvizhi R (2011). Optimal multilevel thresholding using bacterial foraging algorithm, Expert Syst Appl, 38: 15549-64.

Sathya PD, Kayalvizhi. R (2011). Amended bacterial foraging algorithm for multilevel thresholding of magnetic resonance brain images, Measurement, 44: $1828-48$.

Siraj. S (2015). Comparative study of Birge-Massart strategy and unimodalthresholding for image compression using wavelet transform, Optik, 126: 5952-55.

Solmaz A, Farshad T (2017). Detection of brain tumour in 3D MRI images using local binary patterns and histogram orientation gradient. Neurocomputing, 219: 526-35.

Sudip Kumar A, Jamuna KS, Dipak K, Basu MN (2015). Conditional spatial fuzzy C-means clustering algorithm for segmentation of MRI images, Appl Soft Comput, 34: 758-69.

Tahere IM, Mohammad RKM (2009). ECG Compression with Thresholding of 2-D Wavelet Transform Coefficients and Run Length Coding, Eur J Sci Res, 27: 248-57.

Tony FC, Zhou H (2007). Total Variation Wavelet Thresholding, J Sci Comput, 32(2): 315-41. 
Image Anal Stereol 2018;37:249-275

Yudong Z, Lenan W (2011). Optimal multi-level thresholding based on maximum Tsallis entropy via an artificial bee colony approach, Entropy, 13(4): 841-59.

Yuhong L, Fucang J, Jing Q (2016). Brain tumour segmentation from multimodal magnetic resonance images via sparse representation, Artif Intell Med, 73: $1-13$. 